\title{
Hopf algebras and dendriform structures arising from parking functions
}

\author{
by \\ Jean-Christophe Novelli and Jean-Yves Thibon (Marne-la-Vallée)
}

\begin{abstract}
We introduce a graded Hopf algebra based on the set of parking functions (hence of dimension $(n+1)^{n-1}$ in degree $n$ ). This algebra can be embedded into a noncommutative polynomial algebra in infinitely many variables. We determine its structure, and show that it admits natural quotients and subalgebras whose graded components have dimensions respectively given by the Schröder numbers (plane trees), the Catalan numbers, and powers of 3. These smaller algebras are always bialgebras and belong to some family of di- or trialgebras occurring in the works of Loday and Ronco.

Moreover, the fundamental notion of parkization allows one to endow the set of parking functions of fixed length with an associative multiplication (different from the one coming from the Shi arrangement), leading to a generalization of the internal product of symmetric functions. Several of the intermediate algebras are stable under this operation. Among them, one finds the Solomon descent algebra but also a new algebra based on a Catalan set, admitting the Solomon algebra as a left ideal.
\end{abstract}

\section{Contents}

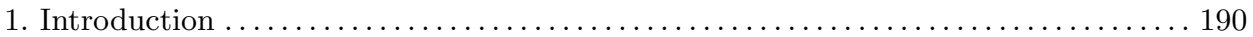

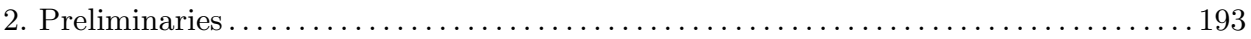

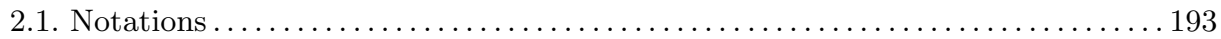

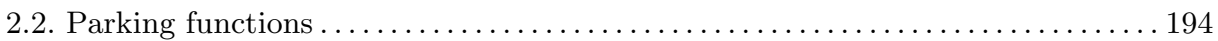

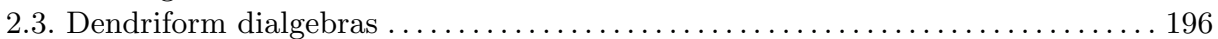

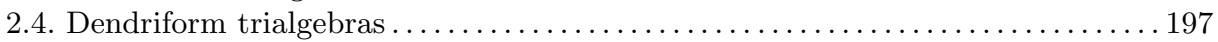

2.5. Bidendriform bialgebras ...................................... 199

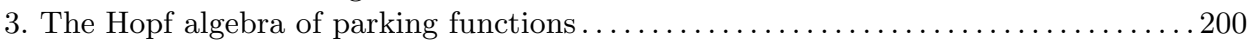

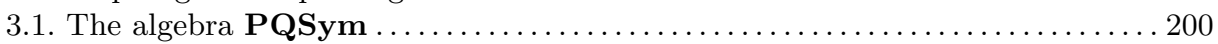

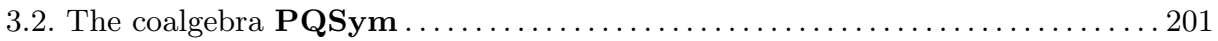

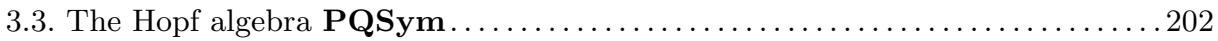

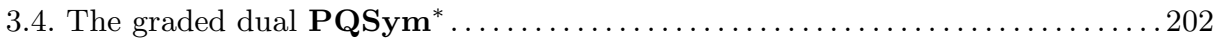

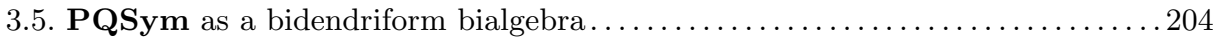

3.6. Free generators and multiplicative bases.......................... 207

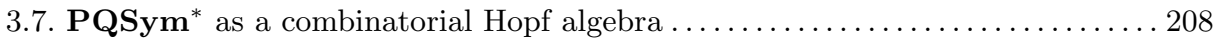

3.8. Realizations of PQSym* and PQSym ........................... 208

2000 Mathematics Subject Classification: Primary 16W30; Secondary $05 E 05$.

Key words and phrases: Hopf algebras, parking functions, symmetric functions. 


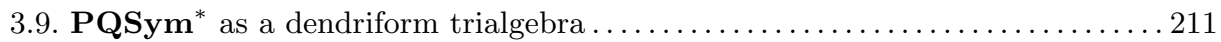

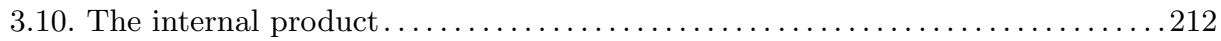

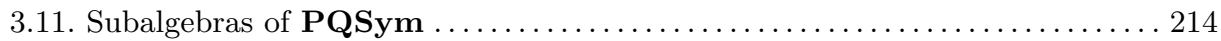

4. The Schröder quasi-symmetric Hopf algebra SQSym ..................... 214

4.1. Hypoplactic classes of parking functions. .......................... 215

4.2. The Schröder quasi-symmetric Hopf algebra SQSym .................. 216

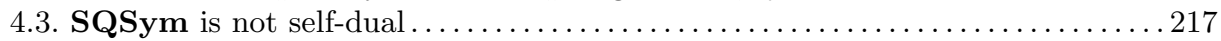

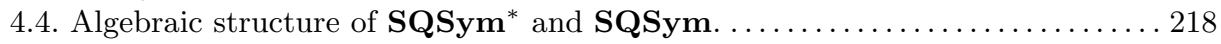

4.5. SQSym* as a combinatorial Hopf algebra............................. 219

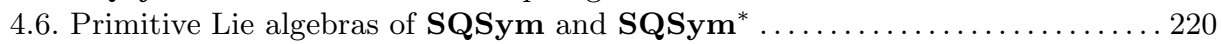

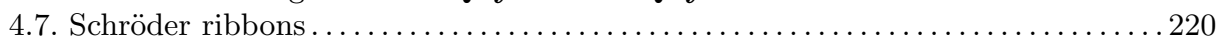

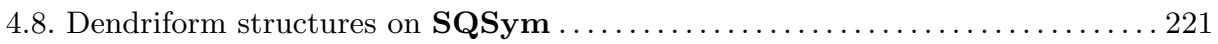

5. The Catalan quasi-symmetric Hopf algebra cQSym ..................... 221

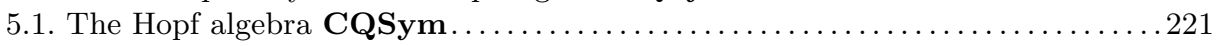

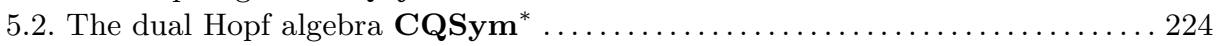

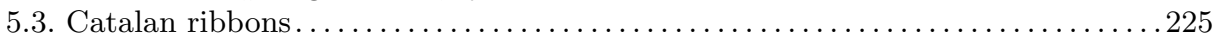

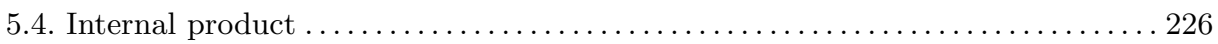

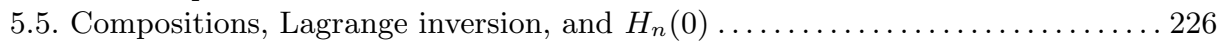

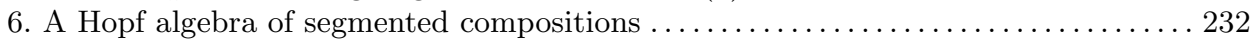

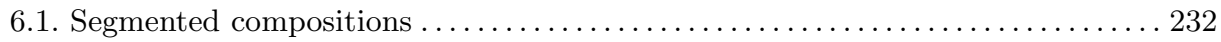

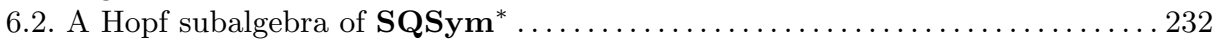

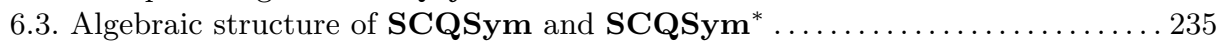

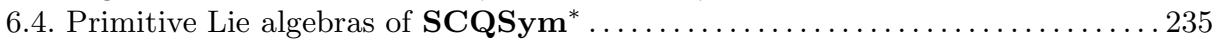

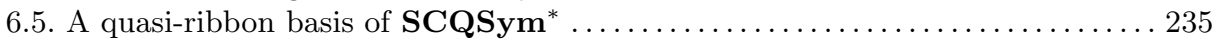

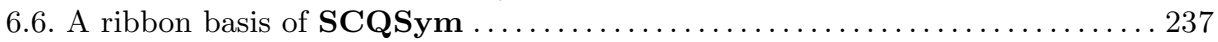

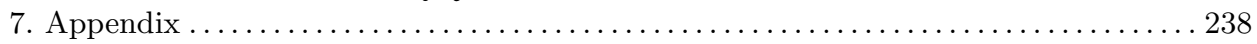

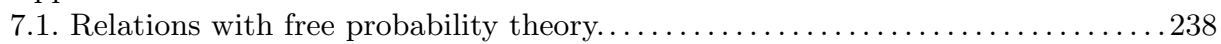

7.2. An exercise on permutation representations ...................... 239

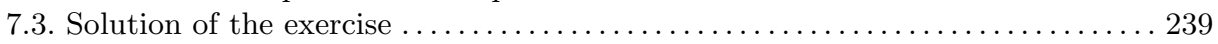

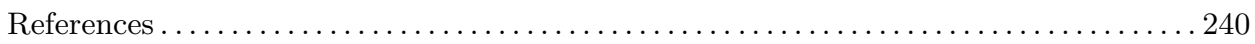

\section{INTRODUCTION}

Many examples of graded Hopf algebras based on combinatorial structures occur in apparently remote contexts. One of them is the theory of operads. It is quite common there that, in a given operad, the free algebra on one generator admits a Hopf structure [15]. This structure often has an elegant combinatorial description, the best known example being the free dendriform algebra on one generator, also known as the Loday-Ronco Hopf algebra of planar binary trees [21, 12], denoted here by PBT.

On the other hand, such Hopf algebras also occur in the theory of noncommutative symmetric functions [7], for which a central problem is to understand complicated commutative formulas by means of simpler noncommutative analogues. It has been found over the years that such an understanding required the introduction of larger and larger Hopf algebras, based on more and more complex combinatorial objects. For such algebras to be 
useful in this context, it is necessary that their elements can be realized as polynomials in some auxiliary infinite set of variables (commutative or not), so as to recover ordinary symmetric functions after a chain of standard manipulations (such as imposing commutation relations among the variables or taking sums to reestablish complete symmetry). The best illustration of this approach is provided by the algebra FQSym of free quasi-symmetric functions [4]. This is an algebra of noncommutative polynomials $\mathbf{F}_{\sigma}(A)$ labelled by permutations. It contains a subalgebra FSym spanned by free Schur functions $\mathbf{S}_{t}(A)$, labelled by standard Young tableaux. This observation essentially amounts to a one-line proof of the Littlewood-Richardson rule. Abstractly, however, FQSym and FSym are isomophic to the Hopf algebras previously introduced by Malvenuto and Reutenauer in [24] and by Poirier and Reutenauer in [32], and it is the polynomial realization which allows such a direct application to symmetric functions.

Interestingly, it is the very same realization which allowed a new understanding of PBT [12]. It could be put on the same footing as FSym, using the sylvester correspondence instead of Robinson-Schensted's, so that both algebras appear now as special cases of a general construction.

The aim of the present article is to introduce a new extension of FQSym, that is, a larger Hopf algebra built from the same principles, but leaving enough room to accomodate several new combinatorial Hopf algebras.

It turns out that most of the Hopf algebras arising in the process also have an operadic interpretation, in general as some kind of trialgebra or dialgebra [19, 22], thus providing polynomial realizations of those as well.

Our master algebra, denoted by PQSym, for parking quasi-symmetric functions, is built on the set of parking functions, a special family of words which can in many respects be regarded as natural generalizations of permutations. Geometrically, permutations correspond to chambers of the Coxeter arrangement of type $A_{n-1}$, while parking functions label those of the Shi arrangement [2]. But this is not the only possible explanation (see, e.g., [20]), and our choice was rather dictated by elementary combinatorial considerations (see Appendix).

Our first task will be to elucidate the structure of PQSym. It will be shown that it is free, cofree, and actually self-dual, with a free primitive Lie algebra. This will be done by means of Foissy's theory of bidendriform bialgebras [6]. Next, we shall determine explicit generators and multiplicative bases of PQSym and PQSym*. Then come the realizations, given by simple and explicit noncommutative polynomials for the natural basis of PQSym*, and in terms of integer matrices, reminiscent of the construction of MQSym [4], for the natural basis of PQSym itself. After that, we shall start the investigation of smaller Hopf algebras arising from PQSym by natural processes. 
Recall that the dimension of PQSym in degree $n$ is $(n+1)^{n-1}$. We shall show that it admits natural quotients and subalgebras whose graded components have dimensions respectively given by the Schröder numbers (plane trees), the Catalan numbers, powers of 3 and powers of 2. Most of those turn out to be related to the theory of operads, and to belong to some family of di- or trialgebras occurring in the works of Loday and Ronco. We shall in particular recover the free dendriform trialgebra (also known as the free tridendriform algebra) on one generator (Schröder numbers) and the free cubical trialgebra. Similarly, we obtain a cocommutative Hopf algebra based on a Catalan set, which is isomorphic to the free dendriform dialgebra on one generator as an algebra, but not as a coalgebra.

Moreover, the fundamental notion of parkization of a word, which is needed from the beginning, allows one to endow the set of parking functions of fixed length with an associative multiplication (different from the one coming from their interpretation as chambers of the Shi arrangement), leading to a generalization of the internal product of symmetric functions. Several of the intermediate algebras are stable under this operation. Among them, one finds the Solomon descent algebra and the Solomon-Tits algebra, but also a new algebra based on a Catalan set, admitting the Solomon algebra as a left ideal.

This paper is structured as follows: the preliminaries present some necessary background about parking functions and dendriform structures and give a realization of the free dendriform trialgebra on one generator in terms of noncommutative polynomials. In Section 3, we present our principal algebra PQSym, and investigate its most important features, mostly relying upon its bidendriform bialgebra structure. We then move to a subalgebra SQSym of PQSym, whose Hilbert series is given by the little Schröder numbers, and prove in particular that it is isomorphic to the free dendriform trialgebra on one generator (Section 4). In Section 5, we study another subalgebra CQSym of PQSym whose Hilbert series is given by the Catalan numbers, show that it is cocommutative, that it is stable under the internal product of PQSym and that its dual is a natural generalization of QSym. In Section 6, we present SCQSym, a quotient of SQSym whose Hilbert series is given by powers of 3 , and show in particular that it is isomorphic to the free cubical trialgebra on one generator. Finally, the Appendix presents how the construction of PQSym arose from considerations about free probability and an exercise proposed by Kerov in 1995. Most of these results were announced in [27].

Acknowledgements. This project has been partially supported by CNRS and by EC's IHRP Programme, grant HPRN-CT-2001-00272, "Algebraic Combinatorics in Europe". The authors would also like to thank 
the contributors of the MuPAD project, and especially those of the combinat package, for providing the development environment for this research (see [14] for an introduction to MuPAD-Combinat).

\section{PRELIMINARIES}

2.1. Notations. Our notations for ordinary symmetric functions will be those of [23]. Other undefined notations can be found in [7, 4], although the essential ones will be recalled when needed.

2.1.1. To start with, we shall need the following two operations on words.

For a word $w$ on the alphabet $\{1,2, \ldots\}$, denote by $w[k]$ the word obtained by replacing each letter $i$ by the integer $i+k$. If $u$ and $v$ are two words, with $u$ of length $k$, one defines the shifted concatenation

$$
u \bullet v=u \cdot(v[k])
$$

and the shifted shuffle

$$
u \uplus v=u \amalg(v[k]) .
$$

where $\amalg$ is the usual shuffle product on words defined recursively by

$$
(a u) \amalg(b v)=a \cdot(u \amalg(b v))+b \cdot((a u) \amalg v),
$$

with $u \amalg \epsilon=\epsilon \amalg u=u$ if $\epsilon$ is the empty word.

It is immediate to see that the set of permutations is closed under both operations. The subalgebra spanned by those elements is isomorphic to the convolution algebra of symmetric groups [24] or to free quasi-symmetric functions [4], whose definition is recalled below.

2.1.2. Let $A$ be a totally ordered alphabet. We denote by $\mathbb{K}$ a field of characteristic 0 , and by $\mathbb{K}\langle A\rangle$ the free associative algebra over $A$ when $A$ is finite, and the projective $\operatorname{limit}_{\operatorname{proj}} \lim _{B} \mathbb{K}\langle B\rangle$, where $B$ runs over finite subsets of $A$, when $A$ is infinite, which will be generally assumed in the following.

Given a totally ordered alphabet $A$, the evaluation vector $\operatorname{Ev}(w)$ of a word $w$ is the sequence of the numbers of occurrences of all the elements of $A$ in $w$.

Recall that the standardization $\operatorname{Std}(w)$ of a word $w \in A^{*}$ is the permutation obtained by iteratively scanning $w$ from left to right, and labelling $1,2, \ldots$ the occurrences of its smallest letter, then numbering the occurrences of the next one, and so on. Alternatively, $\sigma=\operatorname{Std}(w)^{-1}$ can be characterized as the unique permutation of minimal length such that $w \sigma$ is a nondecreasing word. For example, Std(bbacab) $=341625$.

This characterizes completely the sequences of transpositions effected by the bubble sort algorithm on $w$. An elementary observation, which is at the 
basis of the constructions of [4], is that the noncommutative polynomials

$$
\mathbf{G}_{\sigma}(A)=\sum_{w \in A^{*} ; \operatorname{Std}(w)=\sigma} w
$$

where $\sigma$ runs over the set of all permutations, form a basis of a subalgebra of $\mathbb{K}\langle A\rangle$. Moreover, if $A$ is infinite, this subalgebra admits a natural Hopf algebra structure. This is FQSym, the algebra of free quasi-symmetric functions.

Let $\mathbf{F}_{\sigma}=\mathbf{G}_{\sigma^{-1}}$. The coproduct is defined by

$$
\Delta \mathbf{F}_{\sigma}=\sum_{u \cdot v=\sigma} \mathbf{F}_{\operatorname{Std}(u)} \otimes \mathbf{F}_{\operatorname{Std}(v)}
$$

where $u \cdot v$ means concatenation. The scalar product is defined by

$$
\left\langle\mathbf{F}_{\sigma}, \mathbf{G}_{\tau}\right\rangle=\delta_{\sigma, \tau},
$$

where $\delta$ is the Kronecker symbol, and then for all $F, G, H \in$ FQSym one has

$$
\langle F G, H\rangle=\langle F \otimes G, \Delta H\rangle .
$$

The product formula in the $\mathbf{F}$ basis is

$$
\mathbf{F}_{\alpha} \mathbf{F}_{\beta}=\sum_{\gamma \in \alpha \uplus \beta} \mathbf{F}_{\gamma} .
$$

The sum of the inverses of the permutations occurring in $\alpha^{-1} \mathbb{\Psi} \beta^{-1}$ is called convolution and denoted by $\alpha * \beta[33,24]$.

2.1.3. A general process for constructing interesting subalgebras of FQSym is to take sums of the form

$$
\mathcal{P}_{\mathbf{x}}(A)=\sum_{\mathfrak{P}(\sigma)=\mathbf{x}} \mathbf{F}_{\sigma}
$$

where $\mathfrak{P}$ is the left symbol of some Robinson-Schensted type correspondence. If we take the original Robinson-Schensted map, we obtain FSym, the algebra of free symmetric functions [4]. If we take the sylvester congruence [12], we obtain PBT, the Loday-Ronco algebra of planar binary trees. Finally, if we take the hypoplactic correspondence [17], we obtain Sym, the algebra of noncommutative symmetric functions. The dual Hopf algebras are obtained in each case by imposing the corresponding congruence (plactic, sylvester, hypoplactic) on $A^{*}$.

2.2. Parking functions. In the following, we shall see that it is possible to replace permutations by parking functions in all these constructions. It will become obvious that the set of parking functions is stable under shifted concatenation and shifted shuffle, and many other classes of words share this 
property. The point is that for parking functions, the resulting algebra has a natural Hopf structure, and that it is again possible to find a polynomial realization. Moreover, an interesting internal product can be defined.

2.2.1. A parking function is a word $\mathbf{a}=a_{1} \cdots a_{n}$ of length $n$ on the alphabet $[n]=\{1, \ldots, n\}$ whose nondecreasing rearrangement $\mathbf{a}^{\uparrow}=a_{1}^{\prime} \cdots a_{n}^{\prime}$ satisfies $a_{i}^{\prime} \leq i$ for all $i$. Let $\mathrm{PF}_{n}$ be the set of such words.

For example, $\mathrm{PF}_{1}=\{1\}, \mathrm{PF}_{2}=\{11,12,21\}$, and

$$
\begin{aligned}
\mathrm{PF}_{3}= & \{111,112,121,211,113,131,311,122,212,221, \\
& 123,132,213,231,312,321\} .
\end{aligned}
$$

2.2.2. It is well known that $\left|\mathrm{PF}_{n}\right|=(n+1)^{n-1}$, and that the permutation representation of $\mathfrak{S}_{n}$ naturally supported by $\mathrm{PF}_{n}$ has Frobenius characteristic (see [10])

$$
(-1)^{n} \omega\left(h_{n}^{*}\right)
$$

where $f \mapsto f^{*}$ is the involution on symmetric functions defined on the generators $h_{n}$ as follows (see [23, Ex. 24, p. 35]). If we set $H(t):=\sum_{n \geq 0} h_{n} t^{n}$ and $H^{*}(u):=\sum_{n \geq 0} h_{n}^{*} u^{n}$, then

$$
u=t H(t) \Leftrightarrow t=u H^{*}(u) .
$$

Each nondecreasing parking function generates a sub-permutation representation of $\mathrm{PF}_{n}$. It is easy to see that the number of nondecreasing parking functions of length $n$ is the Catalan number $C_{n}=\frac{1}{n+1}\left(\begin{array}{c}2 n \\ n\end{array}\right)$.

2.2.3. Prime parking functions. This important notion has been introduced by Gessel in 1997 (see [37]). Given a parking function of length $n$, one says that $b \in\{0,1, \ldots, n\}$ is a breakpoint of a if $\left|\left\{i \mid a_{i} \leq b\right\}\right|=b$. For example, the parking function 112256679 has the five breakpoints $\{0,4,5,8,9\}$. Then $\mathbf{a} \in \mathrm{PF}_{n}$ is said to be prime if its only breakpoints are the trivial ones: 0 and $n$. Let $\mathrm{PPF}_{n} \subset \mathrm{PF}_{n}$ be the set of prime parking functions on $[n]$. For example,

$$
\mathrm{PPF}_{1}=\{1\}, \quad \mathrm{PPF}_{2}=\{11\}, \quad \mathrm{PPF}_{3}=\{111,112,121,211\} .
$$

It can easily be shown that $\left|\mathrm{PPF}_{n}\right|=(n-1)^{n-1}$ for $n \geq 2$ (see $[37,16]$ and Section 5.5). The number of nondecreasing prime parking functions of length $n$ is the shifted Catalan number $C_{n-1}$ : they are obtained by concatenating a 1 to the left of all nondecreasing parking functions of length $n-1$.

As already mentioned, it is immediate to see that the set of all parking functions is closed under shifted concatenation and shifted shuffle. The prime parking functions are exactly those that do not occur in any nontrivial shifted shuffle of parking functions. This observation is at the basis of our definition of the Hopf algebra of parking functions (see Section 3). 
2.2.4. The module of prime parking functions. Parking functions can be classified according to the factorization of their nondecreasing reorderings $\mathbf{a}^{\uparrow}$ with respect to the operation of shifted concatenation. That is, if

$$
\mathbf{a}^{\uparrow}=w_{1} \bullet \cdots \bullet w_{r}
$$

is the unique maximal factorization of $\mathbf{a}^{\uparrow}$, each $w_{i}$ is a nondecreasing prime parking function. Define $i_{k}=\left|w_{k}\right|$ and let $I=\left(i_{1}, \ldots, i_{r}\right)$. We shall say that $\mathbf{a}$ is of type $I$ and denote by $\mathrm{PPF}_{I}$ the set of parking functions of type $I$. For example, the parking function 966142272 is of type $(1,4,3,1)$ and the number of parking functions of length 4 of each type is

\begin{tabular}{|c|c|c|c|c|c|c|c|}
\hline$(4)$ & $(31)$ & $(13)$ & $(22)$ & $(211)$ & $(121)$ & $(112)$ & $(1111)$ \\
\hline 27 & 16 & 16 & 6 & 12 & 12 & 12 & 24 \\
\hline
\end{tabular}

The set $\mathrm{PPF}_{n}$ of prime parking functions of length $n$ is obviously a sub-permutation representation of $\mathrm{PF}_{n}$. It can be shown that its Frobenius characteristic is

$$
f_{n}=-\omega\left(e_{n}^{*}\right)
$$

(see the Appendix for a direct proof). One can also obtain it as follows.

The set $\mathrm{PPF}_{I}$ of parking functions of type $I$ is a sub-permutation representation of $\mathrm{PF}_{n}$, and its Frobenius characteristic is

$$
\operatorname{ch}\left(\mathrm{PPF}_{I}\right)=f_{i_{1}} \ldots f_{i_{r}},
$$

since it is induced from the permutation representation of the Young subgroup $\mathfrak{S}_{I}$ on the Cartesian product $\mathrm{PPF}_{i_{1}} \times \cdots \times \mathrm{PPF}_{i_{r}}$. Now, $\mathrm{PF}_{n}=$ $\bigsqcup_{I \vDash n} \mathrm{PPF}_{I}$, so that

$$
g_{n}=\sum_{I \models n} f_{i_{1}} \ldots f_{i_{r}}
$$

which amounts to

$$
g:=\sum_{n \geq 0} g_{n}=(1-f)^{-1} \quad \text { where } \quad f=\sum_{n \geq 1} f_{n} .
$$

Thus, if we know that $g_{n}$ is given by (11), we infer that $f_{n}$ is given by (15), and conversely. A noncommutative version of these results will be established in Section 5.5.

2.3. Dendriform dialgebras. A dendriform dialgebra, as defined by Loday [19], is an associative algebra $D$ whose multiplication $\odot$ (it is denoted by $*$ in the original paper) splits into two binary operations

$$
x \odot y=x \ll y+x \gg y,
$$


called left and right, satisfying the following three compatibility relations for all $a, b$, and $c$ different from 1 in $D$ :

$$
\begin{aligned}
& (a \ll b) \ll c=a \ll(b \odot c), \\
& (a \gg b) \ll c=a \gg(b \ll c), \\
& (a \odot b) \gg c=a \gg(b \gg c) .
\end{aligned}
$$

These relations are satisfied by shuffle algebras with $\odot=\Psi$ and for $x=u a$ and $y=v b(a, b \in A)$,

$$
x \gg y=(u a \amalg v) b, \quad x \ll y=(u \amalg v b) a .
$$

It turns out that the free associative algebra $\mathbb{K}\langle A\rangle$ is also a dendriform dialgebra. Actually, it is even a dendriform trialgebra, as explained below.

2.4. Dendriform trialgebras. A dendriform trialgebra [22] is an associative algebra whose multiplication $\odot$ splits into three pieces

$$
x \odot y=x \prec y+x \circ y+x \succ y,
$$

where $\circ$ is associative, and

$$
\begin{aligned}
& (x \prec y) \prec z=x \prec(y \odot z), \\
& (x \succ y) \prec z=x \succ(y \prec z), \\
& (x \odot y) \succ z=x \succ(y \succ z), \\
& (x \succ y) \circ z=x \succ(y \circ z), \\
& (x \prec y) \circ z=x \circ(y \succ z), \\
& (x \circ y) \prec z=x \circ(y \prec z) .
\end{aligned}
$$

Let $A=\left\{a_{1}<a_{2}<\cdots\right\}$ be an infinite linearly ordered alphabet. Recall that $\mathbb{K}\langle A\rangle$ is understood as the projective limit of the $\mathbb{K}\left\langle A_{n}\right\rangle$ where $A_{n}$ is the interval $\left[a_{1}, a_{n}\right]$ of $A$. We denote by $\max (w)$ the greatest letter occurring in the word $w \in A^{*}$.

Definition 2.1. For two nonempty words $u, v \in A^{*}$, we set

$$
\begin{aligned}
& u \prec v= \begin{cases}u v & \text { if } \max (u)>\max (v), \\
0 & \text { otherwise, }\end{cases} \\
& u \circ v= \begin{cases}u v & \text { if } \max (u)=\max (v), \\
0 & \text { otherwise, }\end{cases} \\
& u \succ v= \begin{cases}u v & \text { if } \max (u)<\max (v), \\
0 & \text { otherwise. }\end{cases}
\end{aligned}
$$

LEMMA 2.2. The three operations $\prec, \circ, \succ$, endow the augmentation ideal $\mathbb{K}\langle A\rangle^{+}$with the structure of a dendriform trialgebra.

Proof. A straightforward verification.

Setting $\ll=\prec$ and $\gg=\circ+\succ$, we obtain a dendriform dialgebra. 
It is known [22] that the free dendriform trialgebra on one generator, denoted here by $\mathfrak{T} \mathfrak{D}$, is a free associative algebra with Hilbert series

$$
\begin{aligned}
\sum_{n \geq 0} s_{n} t^{n} & =\frac{1+t-\sqrt{1-6 t+t^{2}}}{4 t} \\
& =1+t+3 t^{2}+11 t^{3}+45 t^{4}+197 t^{5}+\cdots
\end{aligned}
$$

that is, the generating function of the super-Catalan, or little Schröder numbers, counting plane trees.

The previous considerations allow us to give a simple polynomial realization of $\mathfrak{T} \mathfrak{D}$. Consider the polynomial

$$
\mathbf{M}_{1}=\sum_{i \geq 1} a_{i}
$$

(the sum of all letters). We can then state:

THEOREM 2.3. The sub-trialgebra of $\mathbb{K}\langle A\rangle^{+}$generated by $\mathbf{M}_{1}$ is free as a dendriform trialgebra.

We shall need the following construction on words. With any word $w$ of length $n$, associate a plane tree $\mathcal{T}(w)$ with $n+1$ leaves, as follows: if $m=\max (w)$ and if $w$ has exactly $k$ occurrences of $m$, write

$$
w=v_{0} m v_{1} m v_{2} \cdots v_{k-1} m v_{k}
$$

where the $v_{i}$ may be empty. Then $\mathcal{T}(w)$ is the tree obtained by grafting the subtrees $\mathcal{T}\left(v_{0}\right), \mathcal{T}\left(v_{1}\right), \ldots, \mathcal{T}\left(v_{k}\right)$ (in this order) on a common root, with the initial condition $\mathcal{T}(\epsilon)=\emptyset$ for the empty word.

For example, the tree associated with 141324431312 is represented in Figure 1.

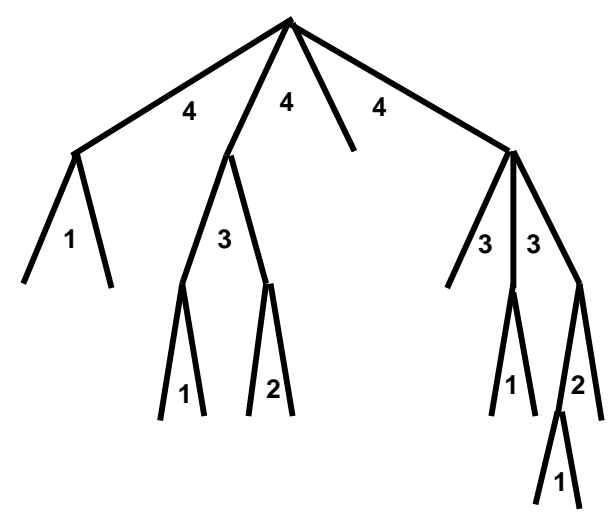

Fig. 1. The tree of 141324431312 
Now associate with each plane tree $T$ a polynomial by

$$
\mathcal{M}_{T}:=\sum_{\mathcal{T}(w)=T} w .
$$

These belong to the subtrialgebra generated by $\mathbf{M}_{1}$, since if $T$ has as subtrees of its root $T_{1}, \ldots, T_{k}$, one has

$$
\mathcal{M}_{T}=\mathcal{M}_{T_{1}} \succ \mathbf{M}_{1} \circ\left(\mathcal{M}_{T_{2}} \succ \mathbf{M}_{1}\right) \circ \cdots \circ\left(\mathcal{M}_{T_{k-1}} \succ \mathbf{M}_{1}\right) \prec \mathbf{M}_{T_{k}} .
$$

For example, for the tree $T$ presented in Figure 1, one gets the expression

$$
\begin{aligned}
\mathbf{M}_{1} & \succ \mathbf{M}_{1} \circ\left(\left(\mathbf{M}_{1} \succ \mathbf{M}_{1} \prec \mathbf{M}_{1}\right) \succ \mathbf{M}_{1}\right) \circ \mathbf{M}_{1} \\
& \prec\left(\mathbf{M}_{1} \circ\left(\mathbf{M}_{1} \succ \mathbf{M}_{1}\right) \prec\left(\mathbf{M}_{1} \succ \mathbf{M}_{1}\right)\right) .
\end{aligned}
$$

Proof of Theorem 2.3. Since it is already known that the dimension of the free dendriform trialgebra on one generator has dimensions given by the little Schröder numbers, we just need to show that all terms of the Hilbert series of this subalgebra are greater than or equal to the terms of (34). The polynomials $\mathcal{M}_{T}$, being sums over disjoint sets of words, are obviously linearly independent, whence the result.

Corollary 2.4 ([22]). The free commutative dendriform trialgebra on one generator is $\mathrm{QSym}^{+}$, the augmentation ideal of quasi-symmetric functions.

Indeed, it is the image of $\mathfrak{T} \mathfrak{D}$ under the ring homomorphism mapping the letters $a_{i}$ to commuting variables $x_{i}$.

Other applications of this realization of $\mathfrak{T D}$ will be given in Section 4 .

2.5. Bidendriform bialgebras. These have been introduced by Foissy in [6]. A bidendriform bialgebra is a dendriform dialgebra equipped with a coproduct that splits into two parts, satisfying the codendriform relations, obtained by dualizing the dendriform relations, and certain compatibility properties with the two half-products.

A codendriform coalgebra is a coalgebra $C$ whose coproduct $\Delta$ splits as $\Delta(c)=\bar{\Delta}(c)+c \otimes 1+1 \otimes c$ and $\bar{\Delta}=\Delta_{\ll}+\Delta_{\gg}$, such that, for all $c$ in $C$ :

$$
\begin{aligned}
\left(\Delta_{\ll} \otimes \operatorname{Id}\right) \circ \Delta_{\ll}(a) & =(\operatorname{Id} \otimes \bar{\Delta}) \circ \Delta_{\ll}(a), \\
\left(\Delta_{\gg} \otimes \operatorname{Id}\right) \circ \Delta_{\ll}(a) & =\left(\operatorname{Id} \otimes \Delta_{\ll}\right) \circ \Delta_{\gg}(a), \\
(\bar{\Delta} \otimes \operatorname{Id}) \circ \Delta_{\gg}(a) & =\left(\operatorname{Id} \otimes \Delta_{\gg}\right) \circ \Delta_{\gg}(a) .
\end{aligned}
$$

The Loday-Ronco algebra of planar binary trees introduced in [21] arises as the free dendriform dialgebra on one generator. This is moreover a Hopf algebra, which turns out to be self-dual, so that it is also codendriform.

There is some compatibility between the dendriform and the codendriform structures, leading to what has been called by Foissy [6] a bidendriform 
bialgebra. A bidendriform bialgebra is both a dendriform dialgebra and a codendriform coalgebra satisfying the following four compatibility relations

$$
\begin{aligned}
\Delta_{\gg}(a \gg b)= & a^{\prime} b_{\gg}^{\prime} \otimes a^{\prime \prime} \gg b_{\gg}^{\prime \prime}+a^{\prime} \otimes a^{\prime \prime} \gg b \\
& +b_{\gg}^{\prime} \otimes a \gg b_{\gg}^{\prime \prime}+a b_{\gg}^{\prime} \otimes b_{\gg}^{\prime \prime}+a \otimes b, \\
\Delta_{\gg}(a \ll b)= & a^{\prime} b_{\gg}^{\prime} \otimes a^{\prime \prime} \ll b_{\gg}^{\prime \prime}+a^{\prime} \otimes a^{\prime \prime} \ll b+b_{\gg}^{\prime} \otimes a \ll b_{\gg}^{\prime \prime}, \\
\Delta_{\ll}(a \gg b)= & a^{\prime} b_{\ll}^{\prime} \otimes a^{\prime \prime} \gg b_{\ll}^{\prime \prime}+a b_{\ll}^{\prime} \otimes b_{\ll}^{\prime \prime}+b_{\ll}^{\prime} \otimes a \gg b_{\ll}^{\prime \prime}, \\
\Delta_{\ll}(a \ll b)= & a^{\prime} b_{\ll}^{\prime} \otimes a^{\prime \prime} \ll b_{\ll}^{\prime \prime}+a^{\prime} b \otimes a^{\prime \prime}+b_{\ll}^{\prime} \otimes a \ll b_{\ll}^{\prime \prime}+b \otimes a,
\end{aligned}
$$

where the pairs $\left(x^{\prime}, x^{\prime \prime}\right)$ (resp. $\left(x_{\ll}^{\prime}, x_{\ll}^{\prime \prime}\right)$ and $\left.\left(x_{\gg}^{\prime}, x_{\gg}^{\prime \prime}\right)\right)$ correspond to all possible elements occurring in $\bar{\Delta} x$ (resp. $\Delta_{\ll} x$ and $\Delta_{\gg} x$ ), summation signs being understood (Sweedler's notation).

Foissy has shown [6] that a connected bidendriform bialgebra $\mathcal{B}$ is always free as an associative algebra and self-dual as a Hopf algebra. Moreover, its primitive Lie algebra is free, and as a dendriform dialgebra, $\mathcal{B}$ is also free over the space of totally primitive elements (those annihilated by $\Delta_{\ll}$ and $\Delta_{\gg}$ ).

It is also proved in [6] that FQSym is bidendriform, so that it has all these properties.

\section{THE HOPF ALGEBRA OF PARKING FUNCTIONS}

3.1. The algebra PQSym. Since permutations are special parking functions and parking functions are stable under the shifted shuffle, it is natural to embed the algebra FQSym of free quasi-symmetric functions of [4] into an algebra spanned by elements $\mathbf{F}_{\mathbf{a}}(\mathbf{a} \in \mathrm{PF})$, with the same multiplication rule:

$$
\mathbf{F}_{\mathbf{a}^{\prime}} \mathbf{F}_{\mathbf{a}^{\prime \prime}}:=\sum_{\mathbf{a} \in \mathbf{a}^{\prime} \cup \mathbf{a}^{\prime \prime}} \mathbf{F}_{\mathbf{a}}
$$

We shall call this algebra PQSym (parking quasi-symmetric functions).

For example,

$$
\begin{aligned}
& \mathbf{F}_{1} \mathbf{F}_{1}=\mathbf{F}_{12}+\mathbf{F}_{21}, \quad \mathbf{F}_{1} \mathbf{F}_{11}=\mathbf{F}_{122}+\mathbf{F}_{212}+\mathbf{F}_{221}, \\
& \mathbf{F}_{1} \mathbf{F}_{12}=\mathbf{F}_{123}+\mathbf{F}_{213}+\mathbf{F}_{231}, \quad \mathbf{F}_{1} \mathbf{F}_{21}=\mathbf{F}_{132}+\mathbf{F}_{312}+\mathbf{F}_{321}, \\
& \mathbf{F}_{12} \mathbf{F}_{11}=\mathbf{F}_{1233}+\mathbf{F}_{1323}+\mathbf{F}_{1332}+\mathbf{F}_{3123}+\mathbf{F}_{3132}+\mathbf{F}_{3312}, \\
& \mathbf{F}_{211} \mathbf{F}_{131}=\mathbf{F}_{211464}+\mathbf{F}_{214164}+\mathbf{F}_{214614}+\mathbf{F}_{214641}+\mathbf{F}_{241164} \\
& +\mathbf{F}_{241614}+\mathbf{F}_{241641}+\mathbf{F}_{246114}+\mathbf{F}_{246141}+\mathbf{F}_{246411} \\
& +\mathbf{F}_{421164}+\mathbf{F}_{421614}+\mathbf{F}_{421641}+\mathbf{F}_{426114}+\mathbf{F}_{426141} \\
& +\mathbf{F}_{426411}+\mathbf{F}_{462114}+\mathbf{F}_{462141}+\mathbf{F}_{462411}+\mathbf{F}_{464211} \text {. }
\end{aligned}
$$

Recall that the prime parking functions are those that do not occur in the decomposition of any nontrivial product $\mathbf{F}_{\mathbf{a}^{\prime}} \mathbf{F}_{\mathbf{a}^{\prime \prime}}$. 
3.2. The coalgebra PQSym. There is a coproduct on PQSym which appears as a natural extension of the coproduct of FQSym. Recall (see $[24,4])$ that if $\sigma$ is a permutation, then

$$
\Delta \mathbf{F}_{\sigma}=\sum_{u \cdot v=\sigma} \mathbf{F}_{\operatorname{Std}(u)} \otimes \mathbf{F}_{\operatorname{Std}(v)}
$$

where Std denotes the usual notion of standardization of a word.

Given a word $w$ on $\{1,2, \ldots\}$, it is possible to define a notion of parkization $\operatorname{Park}(w)$, a parking function which coincides with $\operatorname{Std}(w)$ when $w$ is a word without repeated letters.

Algorithm 3.1. Input: $A$ word $w$.

Output: A parking function.

Let $n$ be the length of $w$. Define

$$
d(w):=\min \left\{i||\left\{w_{j} \leq i\right\} \mid<i\right\} .
$$

- If $d(w)=n+1$, return $w$.

- Otherwise, let $w^{\prime}$ be the word obtained by decreasing by one the values of the elements of $w$ greater than $d(w)$. Then return the parkized word of $w^{\prime}$.

The algorithm is correct since $d(w)=n+1$ iff $w$ is a parking function and since $w^{\prime}$ is smaller than $w$ in the lexicographic order, it terminates.

For example, the following table displays an execution of the parkization algorithm: on each line, there is a word $w$ and the value of $d(w)$ and the next line contains the element $w^{\prime}$ as defined in the algorithm.

\begin{tabular}{cccccccccc}
\hline & \multicolumn{10}{c}{$w$} & & & $d(w)$ \\
\hline 5 & 7 & 3 & 3 & 13 & 1 & 10 & 10 & 4 & 2 \\
4 & 6 & 2 & 2 & 12 & 1 & 9 & 9 & 3 & 7 \\
4 & 6 & 2 & 2 & 11 & 1 & 8 & 8 & 3 & 7 \\
4 & 6 & 2 & 2 & 10 & 1 & 7 & 7 & 3 & 9 \\
4 & 6 & 2 & 2 & 9 & 1 & 7 & 7 & 3 & 10 \\
\hline
\end{tabular}

We can now define a coproduct on PQSym by

$$
\Delta \mathbf{F}_{\mathbf{a}}:=\sum_{u \cdot v=\mathbf{a}} \mathbf{F}_{\operatorname{Park}(u)} \otimes \mathbf{F}_{\operatorname{Park}(v)} .
$$

For example,

$$
\begin{aligned}
& \Delta \mathbf{F}_{121}=1 \otimes \mathbf{F}_{121}+\mathbf{F}_{1} \otimes \mathbf{F}_{21}+\mathbf{F}_{12} \otimes \mathbf{F}_{1}+\mathbf{F}_{121} \otimes 1, \\
& \Delta \mathbf{F}_{131}=1 \otimes \mathbf{F}_{131}+\mathbf{F}_{1} \otimes \mathbf{F}_{21}+\mathbf{F}_{12} \otimes \mathbf{F}_{1}+\mathbf{F}_{131} \otimes 1,
\end{aligned}
$$




$$
\begin{aligned}
\Delta \mathbf{F}_{3132}= & 1 \otimes \mathbf{F}_{3132}+\mathbf{F}_{1} \otimes \mathbf{F}_{132}+\mathbf{F}_{21} \otimes \mathbf{F}_{21} \\
& +\mathbf{F}_{212} \otimes \mathbf{F}_{1}+\mathbf{F}_{3132} \otimes 1 \\
\Delta \mathbf{F}_{1643165}= & 1 \otimes \mathbf{F}_{1643165}+\mathbf{F}_{1} \otimes \mathbf{F}_{532154}+\mathbf{F}_{12} \otimes \mathbf{F}_{32154} \\
& +\mathbf{F}_{132} \otimes \mathbf{F}_{2143}+\mathbf{F}_{1432} \otimes \mathbf{F}_{132}+\mathbf{F}_{15431} \otimes \mathbf{F}_{21} \\
& +\mathbf{F}_{154315} \otimes \mathbf{F}_{1}+\mathbf{F}_{1643165} \otimes 1
\end{aligned}
$$

Proposition 3.2. The operation defined by (54) is coassociative and is a morphism for the product. So (PQSym, $\cdot, \Delta)$ is a bialgebra.

Proof. The operation is obviously coassociative since the deconcatenation is coassociative. Consider two words $w_{1}$ and $w_{2}$ and a prefix $u_{1}$ (resp. $u_{2}$ ) of $w_{1}$ ( of $w_{2}$ ). Then the set of the parkized words of all prefixes of $w_{1} \cup w_{2}$ containing only letters of $u_{1}$ and $u_{2}$ is equal to $u_{1} \cup u_{2}$. So $\Delta$ is a morphism for the product, and hence PQSym is a bialgebra.

3.3. The Hopf algebra PQSym. Since PQSym is endowed with a bialgebra structure naturally graded by the length of parking functions, one defines the antipode as the inverse of the identity for the convolution product and then endows PQSym with a Hopf algebra structure.

The standard formula for the antipode, written in the basis $\left(\mathbf{F}_{\mathbf{a}}\right)$, reads

$$
\nu\left(\mathbf{F}_{\mathbf{a}}\right)=\sum_{r ; u_{1} \cdots u_{r}=\mathbf{a} ;\left|u_{i}\right| \geq 1}(-1)^{r} \mathbf{F}_{\operatorname{Park}\left(u_{1}\right)} \cdots \mathbf{F}_{\operatorname{Park}\left(u_{r}\right)}
$$

For example,

$$
\begin{aligned}
\nu\left(\mathbf{F}_{122}\right) & =-\mathbf{F}_{122}+\mathbf{F}_{1} \mathbf{F}_{11}+\mathbf{F}_{12} \mathbf{F}_{1}-\mathbf{F}_{1}^{3} \\
& =\mathbf{F}_{212}+\mathbf{F}_{221}-\mathbf{F}_{213}-\mathbf{F}_{231}-\mathbf{F}_{321} .
\end{aligned}
$$

3.4. The graded dual PQSym*. Let $\mathbf{G}_{\mathbf{a}}=\mathbf{F}_{\mathbf{a}}^{*} \in \mathbf{P Q S y m}^{*}$ be the dual basis of $\left(\mathbf{F}_{\mathbf{a}}\right)$.

Proposition 3.3. The product on PQSym* is given by

$$
\mathbf{G}_{\mathbf{a}^{\prime}} \mathbf{G}_{\mathbf{a}^{\prime \prime}}=\sum_{\mathbf{a} \in \mathbf{a}^{\prime} *_{P} \mathbf{a}^{\prime \prime}} \mathbf{G}_{\mathbf{a}}
$$

where the convolution $\mathbf{a}^{\prime} *_{P} \mathbf{a}^{\prime \prime}$ of two parking functions is defined as

$$
\mathbf{a}^{\prime} *_{P} \mathbf{a}^{\prime \prime}=\sum_{u, v ; \mathbf{a}=u \cdot v \in \operatorname{PF}, \operatorname{Park}(u)=\mathbf{a}^{\prime}, \operatorname{Park}(v)=\mathbf{a}^{\prime \prime}} \mathbf{a} .
$$

Proof. If $\langle$,$\rangle denotes the duality bracket, the product on PQSym* is$ given by

$$
\mathbf{G}_{\mathbf{a}^{\prime}} \mathbf{G}_{\mathbf{a}^{\prime \prime}}=\sum_{\mathbf{a}}\left\langle\mathbf{G}_{\mathbf{a}^{\prime}} \otimes \mathbf{G}_{\mathbf{a}^{\prime \prime}}, \Delta \mathbf{F}_{\mathbf{a}}\right\rangle \mathbf{G}_{\mathbf{a}}=\sum_{\mathbf{a} \in \mathbf{a}^{\prime} *_{P} \mathbf{a}^{\prime \prime}} \mathbf{G}_{\mathbf{a}}
$$


For example,

$$
\begin{aligned}
\mathbf{G}_{1} \mathbf{G}_{1}= & \mathbf{G}_{11}+\mathbf{G}_{12}+\mathbf{G}_{21}, \\
\mathbf{G}_{1} \mathbf{G}_{11}= & \mathbf{G}_{111}+\mathbf{G}_{122}+\mathbf{G}_{211}+\mathbf{G}_{311}, \\
\mathbf{G}_{1} \mathbf{G}_{12}= & \mathbf{G}_{112}+\mathbf{G}_{113}+\mathbf{G}_{123}+\mathbf{G}_{212}+\mathbf{G}_{213}+\mathbf{G}_{312}, \\
\mathbf{G}_{1} \mathbf{G}_{21}= & \mathbf{G}_{121}+\mathbf{G}_{131}+\mathbf{G}_{132}+\mathbf{G}_{221}+\mathbf{G}_{231}+\mathbf{G}_{321}, \\
\mathbf{G}_{12} \mathbf{G}_{11}= & \mathbf{G}_{1211}+\mathbf{G}_{1222}+\mathbf{G}_{1233}+\mathbf{G}_{1311}+\mathbf{G}_{1322} \\
& +\mathbf{G}_{1411}+\mathbf{G}_{1422}+\mathbf{G}_{2311}+\mathbf{G}_{2411}+\mathbf{G}_{3411}, \\
\mathbf{G}_{211} \mathbf{G}_{131}= & \mathbf{G}_{211131}+\mathbf{G}_{211141}+\mathbf{G}_{211151}+\mathbf{G}_{211161}+\mathbf{G}_{211242} \\
& +\mathbf{G}_{211252}+\mathbf{G}_{211262}+\mathbf{G}_{211353}+\mathbf{G}_{211363}+\mathbf{G}_{211464} \\
& +\mathbf{G}_{322131}+\mathbf{G}_{322141}+\mathbf{G}_{322151}+\mathbf{G}_{322161}+\mathbf{G}_{433141} \\
& +\mathbf{G}_{433151}+\mathbf{G}_{433161}+\mathbf{G}_{433131}+\mathbf{G}_{544131} .
\end{aligned}
$$

When restricted to permutations, the product of $\mathbf{G}$ coincides with the convolution of $[33,24]$. Notice also that

$$
\mathbf{G}_{1}^{n}=\sum_{\mathbf{a} \in \mathrm{PF}_{n}} \mathbf{G}_{\mathbf{a}} .
$$

Proposition 3.4. The coproduct $\Delta \mathbf{G}_{\mathbf{a}}$ is given by

$$
\Delta \mathbf{G}_{\mathbf{a}}:=\sum_{u, v ; \mathbf{a} \in u \uplus v} \mathbf{G}_{u} \otimes \mathbf{G}_{v} .
$$

Proof. If $\langle$,$\rangle denotes the duality bracket, the coproduct on PQSym* is$ given by

$$
\Delta \mathbf{G}_{\mathbf{a}}=\sum_{\mathbf{a}^{\prime}, \mathbf{a}^{\prime \prime}}\left\langle\mathbf{G}_{\mathbf{a}}, \mathbf{F}_{\mathbf{a}^{\prime}} \mathbf{F}_{\mathbf{a}^{\prime \prime}}\right\rangle \mathbf{G}_{\mathbf{a}^{\prime}} \otimes \mathbf{G}_{\mathbf{a}^{\prime \prime}}=\sum_{\mathbf{a} \in \mathbf{a}^{\prime} \cup \mathbf{a}^{\prime \prime}} \mathbf{G}_{\mathbf{a}^{\prime}} \otimes \mathbf{G}_{\mathbf{a}^{\prime \prime}}
$$

For example,

$$
\begin{aligned}
\Delta \mathbf{G}_{121}= & 1 \otimes \mathbf{G}_{121}+\mathbf{G}_{121} \otimes 1 \\
\Delta \mathbf{G}_{131}= & 1 \otimes \mathbf{G}_{131}+\mathbf{G}_{11} \otimes \mathbf{G}_{1}+\mathbf{G}_{131} \otimes 1 \\
\Delta \mathbf{G}_{3132}= & 1 \otimes \mathbf{G}_{3132}+\mathbf{G}_{1} \otimes \mathbf{G}_{221}+\mathbf{G}_{12} \otimes \mathbf{G}_{11}+\mathbf{G}_{3132} \otimes 1 \\
\Delta \mathbf{G}_{164821657}= & 1 \otimes \mathbf{G}_{164821657}+\mathbf{G}_{121} \otimes \mathbf{G}_{315324} \\
& +\mathbf{G}_{1421} \otimes \mathbf{G}_{24213}+\mathbf{G}_{14215} \otimes \mathbf{G}_{1312}+\mathbf{G}_{164821657} \otimes 1 .
\end{aligned}
$$

There is also a direct way to describe the coproduct of $\mathbf{G}_{\mathbf{a}}$ in terms of breakpoints:

Proposition 3.5. Let a be a parking function of length $n$. For $b$ in $\{0, \ldots, n\}$, define $\mathbf{a}^{\prime}(b)$ and $\mathbf{a}^{\prime \prime}(b)$ as the restrictions of $\mathbf{a}$ to the respective intervals $[1, b]$ and $[b+1, n]$. Then

$$
\Delta \mathbf{G}_{\mathbf{a}}:=\sum_{b} \mathbf{G}_{\mathbf{a}^{\prime}(b)} \otimes \mathbf{G}_{\mathbf{a}^{\prime \prime}(b)},
$$

where the sum runs over all breakpoints of $\mathbf{a}$. 
Proof. The term $\mathbf{G}_{u} \otimes \mathbf{G}_{v}$ appears in $\Delta \mathbf{G}_{\mathbf{a}}$ iff a belongs to the shifted shuffle of $u$ and $v$, so that $\mathbf{a}$ has a breakpoint at $b=|u|$. Then $\mathbf{a}^{\prime}(b)=u$ and $\mathbf{a}^{\prime \prime}(b)=v$.

For example, the breakpoints of 164821657 are $\{0,3,4,5,9\}$ so that one recovers the result of (75).

Let us finally mention that the realization provided in Section 3.8.1 allows us to shed an interesting light on the coproduct and the fact that PQSym* is a Hopf algebra.

3.5. PQSym as a bidendriform bialgebra. In [6], Foissy has proved that the Hopf algebra FQSym of free quasi-symmetric functions is bidendriform. A very slight modification of his operations allows us to state:

THEOREM 3.6. PQSym* is a bidendriform bialgebra with the following definitions:

$$
\begin{aligned}
\mathbf{G}_{\mathbf{a}^{\prime}} \ll \mathbf{G}_{\mathbf{a}^{\prime \prime}} & =\sum_{\mathbf{a}=u \cdot v \in \mathbf{a}^{\prime} *_{P} \mathbf{a}^{\prime \prime},|u|=\left|\mathbf{a}^{\prime}\right| ; \max (v)<\max (u)} \mathbf{G}_{\mathbf{a}}, \\
\mathbf{G}_{\mathbf{a}^{\prime}} \gg \mathbf{G}_{\mathbf{a}^{\prime \prime}} & =\sum_{\mathbf{a}=u \cdot v \in \mathbf{a}^{\prime} *_{P} \mathbf{a}^{\prime \prime},|u|=\left|\mathbf{a}^{\prime}\right| ; \max (v) \geq \max (u)} \mathbf{G}_{\mathbf{a}}, \\
\Delta_{\ll} \mathbf{G}_{\mathbf{a}} & =\sum_{\mathbf{a} \in u \uplus v ; \operatorname{last}(\mathbf{a}) \leq|u|} \mathbf{G}_{u} \otimes \mathbf{G}_{v}, \\
\Delta_{\gg} \mathbf{G}_{\mathbf{a}} & =\sum_{\mathbf{a} \in u \uplus v ; \operatorname{last}(\mathbf{a})>|u|} \mathbf{G}_{u} \otimes \mathbf{G}_{v},
\end{aligned}
$$

where $|u| \geq 1$ and $|v| \geq 1$, and last(a) means the last letter of $\mathbf{a}$.

Proof. First, the three defining relations of a dendriform dialgebra are satisfied. Let us check the first one, for instance. The left side of (20) amounts to considering the elements $w$ in $\mathbf{a} *_{P} \mathbf{b} *_{P} \mathbf{c}$ where the last maximum of $w$ belongs to $\mathbf{a}$. It is the same for the right of (20). The other two relations are proved in the same way, by checking that they build the words in $\mathbf{a} *_{P} \mathbf{b} *_{P} \mathbf{c}$ where the last maximum is in $\mathbf{b}$ (equation (21)) or in $\mathbf{c}$ (equation (22)).

The three defining relations of a codendriform coalgebra are also satisfied since they amount to splitting the set of parking functions indexing the elements of $(\bar{\Delta} \otimes \mathrm{Id}) \circ \bar{\Delta}\left(\mathbf{G}_{\mathbf{a}}\right)$ according to the element of the tensor product containing the last letter of $\mathbf{a}$.

Since the sum of the four compatibility relations is equivalent to the coassociativity of $\bar{\Delta}$, it is sufficient to check any three of them. We will only prove the first one (more complicated than the second and third one) in detail, the other ones being proved in the same way.

Till the end of this proof we will identify any function $\mathbf{G}_{\mathbf{a}}$ with its index $\mathbf{a}$. Let $\mathbf{a}$ and $\mathbf{b}$ be two parking functions of length $p$ and $q$. For any word $w$ 
of length $p+q$, let $m_{1}=\min \left(w_{1} \ldots w_{p}\right), m_{2}=\min \left(w_{p+1} \ldots w_{p+q}\right), M_{1}=$ $\max \left(w_{1} \ldots w_{p}\right), M_{2}=\max \left(w_{p+1} \ldots w_{p+q}\right)$.

Let $i$ be any integer and split into two groups and six subgroups the parking functions indexing the terms in $\mathbf{G}_{\mathbf{a}} \gg \mathbf{G}_{\mathbf{b}}$ having a breakpoint at $i$ according to the criterions:

- $\left(M_{1} \leq i\right)$, or $\left(M_{1}>i\right.$ and $\left.m_{1} \leq i\right)$, or $\left(m_{1}>i\right)$,

- $\left(m_{2} \leq i\right)$ or $\left(m_{2}>i\right)$.

Apply $\Delta_{\gg}$ to the first group and let $S_{1}$ be the sum of the elements $\mathbf{G}_{u} \otimes \mathbf{G}_{v}$ such that $u$ is of length $i$. Since $M_{1} \leq i$ and $m_{2} \leq i$, the first $p$ letters of all words are in $u$, whereas the others are both in $u$ and $v$ (by hypothesis, the last one is in $v$ ). Note that the positions of the letters belonging to the right factor of the tensor product are independent of the element of the first group and are the positions of the $p+q-i$ greatest letters of $\mathbf{b}$. Moreover, there exists a breakpoint of $\mathbf{b}$ separating those letters from the other ones. Since the last letter of any word of the first group goes to the right factor of the tensor product and comes from the last letter of $\mathbf{b}$, we then deduce that the right factor of $S_{1}$ is built with all $\mathbf{b}_{\gg}^{\prime \prime}$ of length $p+q-i$, using Sweedler's notations. Finally, in the left factor, we find all the elements of the form $\mathbf{a} \cup \mathbf{b}_{\gg}^{\prime}$, since they correspond to the restriction of all words of $\mathbf{a} \gg \mathbf{b}$ to letters smaller than $i$. Finally, summing up over all possible $i$, we see that the sum of all the elements of all the first groups is $\mathbf{G}_{\mathbf{a}} \mathbf{G}_{\mathbf{b}_{\gg}^{\prime}} \otimes \mathbf{G}_{\mathbf{b}_{\gg}^{\prime \prime}}$, that is, the fourth term of the right-hand side of (43).

In the same way, one proves that the second group, corresponding to $M_{1} \leq i$, and $m_{2}>i$, gives the term $\mathbf{a} \otimes \mathbf{b}$ of (43). The third group, corresponding to $M_{1}>i, m_{1} \leq i$, and $m_{2} \leq i$ gives the term $\mathbf{a}^{\prime} \mathbf{b}_{\gg}^{\prime} \otimes \mathbf{a}^{\prime \prime} \gg \mathbf{b}_{\gg}^{\prime \prime}$ of (43). The fourth group, corresponding to $M_{1}>i, m_{1} \leq i$, and $m_{2}>i$ gives the term $\mathbf{a}^{\prime} \otimes \mathbf{a}^{\prime \prime} \gg \mathbf{b}$ of (43). The fifth group, corresponding to $m_{1}>i$ and $m_{2} \leq i$, gives the term $\mathbf{b}_{\gg}^{\prime} \otimes \mathbf{a} \gg \mathbf{b}_{\gg}^{\prime \prime}$ of (43). The sixth group, corresponding to $m_{1}>i$ and $m_{2}>i$, gives no term since we would have $|u|=0$, which is impossible.

For example,

$$
\begin{aligned}
\mathbf{G}_{12} \ll \mathbf{G}_{212}= & \mathbf{G}_{13212}+\mathbf{G}_{14212}+\mathbf{G}_{14313}+\mathbf{G}_{14323}+\mathbf{G}_{15212} \\
& +\mathbf{G}_{15313}+\mathbf{G}_{15323}+\mathbf{G}_{24313}+\mathbf{G}_{24212}+\mathbf{G}_{34212} \\
& +\mathbf{G}_{23212}+\mathbf{G}_{25212}+\mathbf{G}_{25313}+\mathbf{G}_{35212}+\mathbf{G}_{45212}, \\
\mathbf{G}_{12} \gg \mathbf{G}_{212}= & \mathbf{G}_{12212}+\mathbf{G}_{12313}+\mathbf{G}_{12323}+\mathbf{G}_{12414}+\mathbf{G}_{12424} \\
& +\mathbf{G}_{12434}+\mathbf{G}_{13313}+\mathbf{G}_{13323}+\mathbf{G}_{13414}+\mathbf{G}_{13424} \\
& +\mathbf{G}_{23313}+\mathbf{G}_{23414}, \\
\Delta_{\ll} \mathbf{G}_{1252754}= & \mathbf{G}_{125254} \otimes \mathbf{G}_{1}+\mathbf{G}_{1224} \otimes \mathbf{G}_{131}, \\
\Delta_{\gg} \mathbf{G}_{1252754}= & \mathbf{G}_{122} \otimes \mathbf{G}_{2421}+\mathbf{G}_{1} \otimes \mathbf{G}_{141643} .
\end{aligned}
$$


The duality of bidendriform bialgebras implies that the bidendriform relations for PQSym are

$$
\begin{gathered}
\mathbf{F}_{\mathbf{a}^{\prime}} \ll \mathbf{F}_{\mathbf{a}^{\prime \prime}}=\sum_{\mathbf{a} \in \mathbf{a}^{\prime} \uplus \mathbf{a}^{\prime \prime} ; \operatorname{last}(\mathbf{a}) \leq\left|\mathbf{a}^{\prime}\right|} \mathbf{F}_{\mathbf{a}}, \\
\mathbf{F}_{\mathbf{a}^{\prime}} \gg \mathbf{F}_{\mathbf{a}^{\prime \prime}}=\sum_{\mathbf{a} \in \mathbf{a}^{\prime} \uplus \mathbf{a}^{\prime \prime} ; \operatorname{last}(\mathbf{a})>\left|\mathbf{a}^{\prime}\right|} \mathbf{F}_{\mathbf{a}}, \\
\Delta_{\ll} \mathbf{F}_{\mathbf{a}}=\sum_{u \cdot v=\mathbf{a} ; \max (v)<\max (u)} \mathbf{F}_{\operatorname{Park}(u)} \otimes \mathbf{F}_{\operatorname{Park}(v),}, \mathbf{F}_{\operatorname{Park}(u)} \otimes \mathbf{F}_{\operatorname{Park}(v)},
\end{gathered}
$$

where the sums inside the coproducts range over nontrivial deconcatenations, that is, $|u| \geq 1$ and $|v| \geq 1$.

We then have the following consequences of the results of Foissy [6].

Corollary 3.7. PQSym is a self-dual Hopf algebra.

Corollary 3.8. The Lie algebra of primitive elements of PQSym is a free Lie algebra (its generators are not explicitly known).

Let

$$
P F(t)=1+\sum_{n \geq 1}(n+1)^{n-1} t^{n}
$$

Corollary 3.9. PQSym is free as a dendriform dialgebra on its totally primitive elements whose degree generating series is

$$
\begin{aligned}
T P(t):= & \frac{P F(t)-1}{P F(t)^{2}} \\
= & t+t^{2}+7 t^{3}+66 t^{4}+786 t^{5}+11278 t^{6}+189391 t^{7} \\
& +3648711 t^{8}+79447316 t^{9}+O\left(t^{10}\right) .
\end{aligned}
$$

For example, $\mathbf{F}_{1}$ and $\mathbf{G}_{1}$ are totally primitive and so are $\mathbf{F}_{12}-\mathbf{F}_{11}$ and $\mathbf{G}_{11}$. Here are bases of the seven-dimensional space of totally primitive elements of PQSym and PQSym* in degree 3:

$$
\begin{gathered}
\mathbf{F}_{123}-\mathbf{F}_{122}-\mathbf{F}_{112}+\mathbf{F}_{111}, \quad \mathbf{F}_{311}-\mathbf{F}_{211}, \quad \mathbf{F}_{113}-\mathbf{F}_{112}, \\
\mathbf{F}_{131}-\mathbf{F}_{121}, \quad \mathbf{F}_{132}-\mathbf{F}_{131}, \quad \mathbf{F}_{231}-\mathbf{F}_{121}, \quad \mathbf{F}_{213}-\mathbf{F}_{212} ; \\
\mathbf{G}_{122}-\mathbf{G}_{212}, \quad \mathbf{G}_{131}-\mathbf{G}_{311}, \quad \mathbf{G}_{312}-\mathbf{G}_{132}, \\
\mathbf{G}_{111}, \quad \mathbf{G}_{112}, \quad \mathbf{G}_{121}, \quad \mathbf{G}_{211} .
\end{gathered}
$$

Thanks to the bidendriform structure of PQSym, we know that PQSym and PQSym* are isomorphic as bidendriform bialgebras and hence 
isomorphic as Hopf algebras. We do not know an explicit isomorphism, but restricting to FQSym, that is, permutations, the linear map $\varphi$ defined by

$$
\varphi\left(\mathbf{F}_{\sigma}\right):=\sum_{\mathbf{a}, \operatorname{Std}(\mathbf{a})=\sigma^{-1}} \mathbf{G}_{\mathbf{a}}
$$

is a bidendriform and hence a Hopf embedding, compatible with the usual realization of FQSym [4].

3.6. Free generators and multiplicative bases. Let us say that a word $w$ over $\mathbb{N}^{*}$ is connected if it cannot be written as a shifted concatenation $w=u \bullet v$, and anti-connected if its mirror image $\bar{w}$ is connected.

Proposition 3.10. PQSym is free over the set

$$
\left\{\mathbf{F}_{\mathbf{c}} \mid \mathbf{c} \in \mathrm{PF}, \mathbf{c} \text { connected }\right\}
$$

and PQSym* is free over the set

$$
\left\{\mathbf{G}_{\mathbf{d}} \mid \mathbf{d} \in \mathrm{PF}, \mathbf{d} \text { anti-connected }\right\} .
$$

Proof. Clearly, any word $w$ has a unique maximal factorization into connected words, $w=w_{1} \bullet \cdots \bullet w_{k}$ where all $w_{i}$ are connected. Moreover, the lexicographically minimal word in $w_{1} \cup \ldots \cup w_{k}$ is $w$ so that the matrix expressing all products of $\mathbf{F}$ indexed by connected words is triangular over the basis $\mathbf{F}_{\mathbf{a}}$, with ones on the diagonal. The proof is exactly the same for the $\mathbf{G}$.

The ordinary generating function for the numbers $c_{n}$ of connected parking functions is

$$
\begin{aligned}
\sum_{n \geq 1} c_{n} t^{n}=1 & -P F(t)^{-1} \\
= & t+2 t^{2}+11 t^{3}+92 t^{4}+1014 t^{5}+13795 t^{6}+223061 t^{7} \\
& +4180785 t^{8}+89191196 t^{9}+2135610879 t^{10} \\
& +56749806356 t^{11}+1658094051392 t^{12}+O\left(t^{13}\right)
\end{aligned}
$$

Let $\mathbf{a}=\mathbf{a}_{1} \bullet \cdots \bullet \mathbf{a}_{r}$ be the maximal factorization of $\mathbf{a}$ into connected parking functions. We set

$$
\begin{aligned}
\mathbf{F}^{\mathbf{a}} & =\mathbf{F}_{\mathbf{a}_{1}} \cdots \mathbf{F}_{\mathbf{a}_{r}}, \\
\mathbf{G}^{\overline{\mathbf{a}}} & =\mathbf{G}_{\overline{\mathbf{a}}_{r}} \cdots \mathbf{G}_{\overline{\mathbf{a}}_{1}} .
\end{aligned}
$$

Proposition 3.11. The basis ( $\left.\mathbf{F}^{\mathbf{a}}\right)$ of $\mathbf{P Q S y m}$ and the basis $\left(\mathbf{G}^{\overline{\mathbf{a}}}\right)$ of PQSym* are both multiplicative.

Proof. This follows from the proof of Proposition 3.10. 
Now, if $\mathbf{S}_{\mathbf{a}}$ (resp. $\mathbf{T}_{\mathbf{a}}$ ) is the dual basis of $\mathbf{F}^{\mathbf{a}}\left(\right.$ resp. $\left.\mathbf{G}^{\overline{\mathbf{a}}}\right)$ then $\left\{\mathbf{S}_{\mathbf{c}} \mid \mathbf{c}\right.$ connected $\}$ and $\left\{\mathbf{T}_{\mathbf{c}} \mid \mathbf{c}\right.$ connected $\}$

are bases of the primitive Lie algebras $\mathbf{L P Q}{ }^{*}$ (resp. LPQ) of PQSym* (resp. PQSym).

Thanks again to [6], we know that both Lie algebras are free, on generators whose degree generating series is

$$
\begin{aligned}
1-\prod_{n \geq 1}( & \left.-t^{n}\right)^{c_{n}}=1-(1-t)\left(1-t^{2}\right)^{2}\left(1-t^{3}\right)^{11} \cdots \\
& =t+2 t^{2}+9 t^{3}+80 t^{4}+901 t^{5}+12564 t^{6}+206476 t^{7} \\
& +3918025 t^{8}+84365187 t^{9}+2034559143 t^{10}+O\left(t^{11}\right) .
\end{aligned}
$$

3.7. PQSym* as a combinatorial Hopf algebra. Since FQSym can be embedded in PQSym, we have a canonical Hopf embedding of Sym in PQSym given by

$$
S_{n} \mapsto \mathbf{F}_{12 \cdots n} .
$$

With parking functions, we have other possibilities: for example,

$$
j\left(S_{n}\right):=\mathbf{F}_{11 \cdots 1}
$$

is a Hopf embedding, whose dual $j^{*}$ maps PQSym* to QSym and therefore endows PQSym* with a different structure of combinatorial Hopf algebra in the sense of [1].

On the dual side, we have a Hopf embedding

$$
S_{n} \mapsto \sum_{\operatorname{Std}(\mathbf{a})=12 \cdots n} \mathbf{G}_{\mathbf{a}}
$$

of Sym into PQSym*, given by the restriction of the self-duality isomorphism of (93) to the Sym subalgebra $S_{n}=\mathbf{F}_{12 \cdots n}$ of PQSym. Its transpose gives a Hopf epimorphism $\eta: \mathbf{P Q S y m} \rightarrow$ QSym, which maps $\mathbf{F}_{\mathbf{a}}$ to $F_{I}$, where $I$ is the descent composition of the word $\mathbf{a}$.

\subsection{Realizations of PQSym* and PQSym}

3.8.1. Realization of PQSym*. The algebra $\mathbf{P Q S y m}{ }^{*}(A)$ admits a simple realization in terms of noncommutative polynomials [28], which is similar to the construction of FQSym. If $A$ is a totally ordered infinite alphabet, one can set

$$
\mathbf{G}_{\mathbf{a}}(A):=\sum_{w \in A^{*}, \operatorname{Park}(w)=\mathbf{a}} w .
$$


THEOREM $3.12([28])$. These polynomials satisfy (61) and allow writing the coproduct as $\Delta \mathbf{G}_{\mathbf{a}}=\mathbf{G}_{\mathbf{a}}\left(A^{\prime} \hat{+} A^{\prime \prime}\right)$ where $A^{\prime} \hat{+} A^{\prime \prime}$ denotes the ordered sum of two mutually commuting alphabets isomorphic to $A$ as ordered sets.

Let us recall the precise way to introduce a coproduct on an algebra realized on words under certain conditions. Start with $A^{\prime}$ and $A^{\prime \prime}$, two mutually commuting alphabets isomorphic to $A$ as ordered sets. Then build their ordered sum $A^{\prime} \hat{+} A^{\prime \prime}$ and compute $\mathbf{G}_{\mathbf{a}}\left(A^{\prime} \hat{+} A^{\prime \prime}\right)$ separating inside each term what belongs to $A^{\prime}$ and what belongs to $A^{\prime \prime}$. Assume that one can write, for all parking functions $\mathbf{a}$,

$$
\mathbf{G}_{\mathbf{a}}\left(A^{\prime} \hat{+} A^{\prime \prime}\right)=\sum_{\mathbf{a}^{\prime}, \mathbf{a}^{\prime \prime}} \mathbf{G}_{\mathbf{a}^{\prime}}\left(A^{\prime}\right) \mathbf{G}_{\mathbf{a}^{\prime \prime}}\left(A^{\prime \prime}\right),
$$

where the sum is taken over a set of pairs of parking functions depending on a. Then the operation

$$
\Delta \mathbf{G}_{\mathbf{a}}:=\sum_{\mathbf{a}^{\prime}, \mathbf{a}^{\prime \prime}} \mathbf{G}_{\mathbf{a}^{\prime}} \otimes \mathbf{G}_{\mathbf{a}^{\prime \prime}},
$$

where the sum is taken over the same set as before, is a coproduct.

For example, $\mathbf{G}_{121}=\sum_{i} a_{i} a_{i+1} a_{i}$, so that

$$
\mathbf{G}_{121}\left(A^{\prime} \hat{+} A^{\prime \prime}\right)=\mathbf{G}_{121}\left(A^{\prime}\right)+\mathbf{G}_{121}\left(A^{\prime \prime}\right),
$$

since $a_{i} \in A^{\prime}$ is equivalent to $a_{i+1} \in A^{\prime}$ by definition of the ordered sum of alphabets. One then recovers the results of (72).

Now, $\mathbf{G}_{131}=\sum_{i, j ; j>i+1} a_{i} a_{j} a_{i}$, so that

$$
\mathbf{G}_{131}\left(A^{\prime} \hat{+} A^{\prime \prime}\right)=\mathbf{G}_{131}\left(A^{\prime}\right)+\mathbf{G}_{11}\left(A^{\prime}\right) \mathbf{G}_{1}\left(A^{\prime \prime}\right)+\mathbf{G}_{121}\left(A^{\prime \prime}\right),
$$

since $a_{i}$ and $a_{j}$ can belong to $A$ (first term), or $a_{i}$ belongs to $A^{\prime}$ and $a_{j}$ belongs to $A^{\prime \prime}$ (second term), or $a_{i}$ and $a_{j}$ belong to $A^{\prime \prime}$ (third term). One then recovers the results of (73).

3.8.2. Realization of PQSym. Although PQSym and PQSym* are isomorphic as Hopf algebras, no explicit isomorphism is known. We can nevertheless propose a realization of PQSym in terms of $(0,1)$-matrices instead of words.

This construction is reminiscent of the construction of MQSym (see $[11,4]$ ), and coincides with it when restricted to permutation matrices, providing the natural embedding of FQSym in MQSym.

Let $\mathcal{M}_{n}$ be the vector space spanned by symbols $X_{M}$ where $M$ runs over $(0,1)$-matrices with $n$ columns and an infinite number of rows, with $n$ nonzero entries, so that at most $n$ rows are nonzero.

Given such a matrix $M$, we define its vertical packing $\operatorname{vp}(M)$ as the finite matrix obtained by removing the null rows of $M$. 
For a vertically packed matrix $P$, we define

$$
\mathbf{M}_{P}=\sum_{\mathrm{vp}(M)=P} X_{M}
$$

Now, given a $(0,1)$-matrix, we define its reading $r(M)$ as the word obtained by reading its entries row-wise, from left to right and from top to bottom and recording the numbers of the columns of the ones. For example, the reading of the matrix

$$
\left(\begin{array}{llll}
0 & 1 & 1 & 0 \\
1 & 0 & 0 & 0 \\
0 & 1 & 0 & 0
\end{array}\right)
$$

is $(2,3,1,2)$.

A matrix $M$ is said to be of parking type if $r(M)$ is a parking function. Finally, for a parking function a, we set

$$
\mathbf{F}_{\mathbf{a}}:=\sum_{r(P)=\mathbf{a}, P \text { vertically packed }} \mathbf{M}_{P}=\sum_{r(M)=\mathbf{a}} X_{M} .
$$

For example,

$$
\mathbf{F}_{(1,2,2)}=\mathbf{M}_{\left(\begin{array}{lll}
1 & 1 & 0 \\
0 & 1 & 0
\end{array}\right)}+\mathbf{M}_{\left(\begin{array}{lll}
1 & 0 & 0 \\
0 & 1 & 0 \\
0 & 1 & 0
\end{array}\right) .}
$$

The multiplication on $\mathcal{M}=\bigoplus_{n} \mathcal{M}_{n}$ is defined by columnwise concatenation of matrices:

$$
X_{M} X_{N}=X_{M \cdot N}
$$

In order to decribe explicitly the product of $\mathbf{M}_{P}$ and $\mathbf{M}_{Q}$, we first need a definition. Let $P$ and $Q$ be two vertically packed matrices with respective heights $p$ and $q$. The augmented shuffle of $P$ and $Q$ is defined as follows: let $r$ be an integer in $[\max (p, q), p+q]$. One inserts zero rows in $P$ and $Q$ in all possible ways so that the resulting matrices have $p+q$ rows. Let $R$ be the matrix obtained by concatenation of such pairs of matrices. The augmented shuffle is the set of such matrices $R$ with nonzero rows. We denote this set by $\uplus(P, Q)$.

THEOREM 3.13. The following formulas hold:

$$
\begin{aligned}
\mathbf{M}_{P} \mathbf{M}_{Q} & =\sum_{R \in \uplus(P, Q)} \mathbf{M}_{R}, \\
\mathbf{F}_{\mathbf{a}^{\prime}} \mathbf{F}_{\mathbf{a}^{\prime \prime}} & =\sum_{\mathbf{a} \in \mathbf{a}^{\prime} \uplus \mathbf{a}^{\prime \prime}} \mathbf{F}_{\mathbf{a}},
\end{aligned}
$$

This is the same as equation (47). 
Proof. Formula (114) comes from the definition of the augmented shuffle of matrices: any matrix in $\uplus(P, Q)$ appears as a product $X_{M} \cdot X_{N}$ where $\operatorname{vp}(M)=P$ and $\operatorname{vp}(N)=Q$. Conversely, any element in $\mathbf{M}_{P} \mathbf{M}_{Q}$ has as vertical packing a matrix whose number of rows is in the interval $[\max (p, q), p+q]$ and whose left part has $M$ as vertical packing and whose right part has $N$ as vertical packing.

The proof of (115) is almost the same as the previous one if one starts from the definition $\mathbf{F}_{\mathbf{a}}=\sum_{r(M)=\mathbf{a}} X_{M}$.

Finally, concerning the coproduct, one has first to define the parkization $\operatorname{Park}(M)$ of a vertically packed matrix $M$, which consists in iteratively removing column $d(r(M))$ until $M$ becomes a parking matrix.

The coproduct of a matrix $\mathbf{M}_{P}$ is then defined as

$$
\Delta \mathbf{M}_{P}=\sum_{Q \cdot R=P} \mathbf{M}_{\operatorname{Park}(Q)} \otimes \mathbf{M}_{\operatorname{Park}(R)} .
$$

It is then easy to check that

Proposition 3.14. The following formula holds:

$$
\Delta \mathbf{F}_{\mathbf{a}}=\sum_{u \cdot v=\mathbf{a}} \mathbf{F}_{\operatorname{Park}(u)} \otimes \mathbf{F}_{\operatorname{Park}(v)} .
$$

This is the same as equation (52).

3.8.3. Realization of FQSym. A parking matrix $M$ is said to be a word matrix if there is exactly one 1 in each column. Then FQSym is the Hopf subalgebra generated by the parking word matrices.

3.9. PQSym* as a dendriform trialgebra. Since we already know that $\mathbb{K}\langle A\rangle^{+}$is a dendriform trialgebra (see Definition 2.1 and Lemma 2.2), and since PQSym* can be realized on words, it is a natural question to ask whether PQSym* is a sub-trialgebra of $\mathbb{K}\langle A\rangle^{+}$.

THEOREM 3.15. PQSym* is a sub-dendriform trialgebra of $\mathbb{K}\langle A\rangle^{+}$with the following product rules:

$$
\begin{aligned}
\mathbf{G}_{\mathbf{a}^{\prime}} \prec \mathbf{G}_{\mathbf{a}^{\prime \prime}} & =\sum_{\mathbf{a}=u \cdot v \in \mathbf{a}^{\prime} *_{P} \mathbf{a}^{\prime \prime},|u|=\left|\mathbf{a}^{\prime}\right| ; \max (v)<\max (u)} \mathbf{G}_{\mathbf{a}}, \\
\mathbf{G}_{\mathbf{a}^{\prime}} \circ \mathbf{G}_{\mathbf{a}^{\prime \prime}} & =\sum_{\mathbf{a}=u \cdot v \in \mathbf{a}^{\prime} *_{P} \mathbf{a}^{\prime \prime},|u|=\left|\mathbf{a}^{\prime}\right| ; \max (v)=\max (u)} \mathbf{G}_{\mathbf{a}}, \\
\mathbf{G}_{\mathbf{a}^{\prime}} \succ \mathbf{G}_{\mathbf{a}^{\prime \prime}} & =\sum_{\mathbf{a}=u \cdot v \in \mathbf{a}^{\prime} *_{P} \mathbf{a}^{\prime \prime},|u|=\left|\mathbf{a}^{\prime}\right| ; \max (v)>\max (u)} \mathbf{G}_{\mathbf{a}} .
\end{aligned}
$$

Proof. Since PQSym* can be realized on words, one only needs to check that PQSym* is stable under all three operations, their compatibility coming from the fact that $\mathbb{K}\langle A\rangle^{+}$is a dendriform trialgebra. Since all words 
having a given parkized word have the same inversions, they have in particular the same relations between the maximum of any prefix and any suffix of given lengths. One then derives the product rules from direct calculation.

For example, with the notations of Definition 2.1:

$$
\begin{aligned}
\mathbf{G}_{12} \prec \mathbf{G}_{212}= & \mathbf{G}_{13212}+\mathbf{G}_{14212}+\mathbf{G}_{14313}+\mathbf{G}_{14323}+\mathbf{G}_{15212} \\
& +\mathbf{G}_{15313}+\mathbf{G}_{15323}+\mathbf{G}_{24313}+\mathbf{G}_{24212}+\mathbf{G}_{34212} \\
& +\mathbf{G}_{23212}+\mathbf{G}_{25212}+\mathbf{G}_{25313}+\mathbf{G}_{35212}+\mathbf{G}_{45212}, \\
\mathbf{G}_{12} \circ \mathbf{G}_{212}= & \mathbf{G}_{12212}+\mathbf{G}_{13313}+\mathbf{G}_{13323}+\mathbf{G}_{23313}, \\
\mathbf{G}_{12} \succ \mathbf{G}_{212}= & \mathbf{G}_{12313}+\mathbf{G}_{12323}+\mathbf{G}_{12414}+\mathbf{G}_{12424} \\
& +\mathbf{G}_{12434}+\mathbf{G}_{13414}+\mathbf{G}_{13424}+\mathbf{G}_{23414} .
\end{aligned}
$$

Based on numerical evidence, we conjecture the following result:

Conjecture 3.16. PQSym* is a free dendriform trialgebra.

Recall that the generating series $F(t)$ for the dimensions of the free dendriform trialgebra satisfies

$$
F(t)-1=t\left(2 F(t)^{2}-F(t)\right) .
$$

Applying the same trick as in [6] for computing the generating series of the totally primitive elements, one gets the generating series of the number $g_{n}$ of generators in degree $n$ of $\mathbf{P Q S y m}{ }^{*}$ as a free dendriform trialgebra:

$$
\begin{aligned}
\sum_{n \geq 0} g_{n} t^{n}= & \frac{P F(t)-1}{2 P F(t)^{2}-P F(t)} \\
= & t+5 t^{3}+50 t^{4}+634 t^{5}+9475 t^{6}+163843 t^{7} \\
& +3226213 t^{8}+71430404 t^{9}+O\left(t^{10}\right) .
\end{aligned}
$$

By self-duality of PQSym, one can endow PQSym with a structure of dendriform trialgebra.

Note that FQSym is not a sub-dendriform trialgebra of $\mathbb{K}\langle A\rangle^{+}$since the product is not internal and that, independently of the realization, it cannot be a free dendriform trialgebra since the substitution $F(t)=\sum_{n} n ! t^{n}$ in (125) does not yield a series with nonnegative integer coefficients.

3.10. The internal product. We shall now recall the definition of the internal product of PQSym, introduced in [28]. We first need a few standard notations about biwords. Let $x_{i j}=\left(\begin{array}{l}i \\ j\end{array}\right)$ be commuting indeterminates, and $a_{i j}=\left[\begin{array}{l}i \\ j\end{array}\right]$ be noncommuting ones. We shall denote by $\left(\begin{array}{l}i_{1} \cdots i_{r} \\ j_{1} \cdots j_{r}\end{array}\right)$ the monomial $\left(\begin{array}{l}i_{1} \\ j_{1}\end{array}\right) \cdots\left(\begin{array}{l}i_{1} \\ j_{1}\end{array}\right)$ and by $\left[\begin{array}{l}i_{1} \cdots i_{r} \\ j_{1} \cdots j_{r}\end{array}\right]$ the word $\left[\begin{array}{l}i_{1} \\ j_{1}\end{array}\right] \cdots\left[\begin{array}{l}i_{1} \\ j_{1}\end{array}\right]$. Such expressions will be referred to respectively as bimonomials and biwords. 
Recall that Gessel constructed the descent algebra by extending to QSym the coproduct dual to the internal product of symmetric functions. That is, if $X$ and $Y$ are two totally and isomorphically ordered alphabets of commuting variables, we can identify a tensor product $f \otimes g$ of quasi-symmetric functions with $f(X) g(Y)$. Denoting by $X Y$ the Cartesian product $X \times Y$ endowed with the lexicographic order, Gessel defined, for $f \in Q S y m_{n}$,

$$
\delta(f)=f(X Y) \in \text { QSym }_{n} \otimes Q \text { Sym }_{n} .
$$

The dual operation on $\mathbf{S y m}_{n}$ is the internal product $*$, for which it is antiisomorphic to the descent algebra $\Sigma_{n}$. This construction can be extended to PQSym*. Let $A^{\prime}$ and $A^{\prime \prime}$ be two totally and isomorphically ordered alphabets of noncommuting variables, but such that $A^{\prime}$ and $A^{\prime \prime}$ commute with each other. We denote by $A^{\prime} A^{\prime \prime}$ the Cartesian product $A^{\prime} \times A^{\prime \prime}$ endowed with the lexicographic order. This is a total order in which each element has a successor, so that $G_{\mathbf{a}}\left(A^{\prime} A^{\prime \prime}\right)$ is a well defined polynomial. Identifying tensor products of words of the same length with words over $A^{\prime} A^{\prime \prime}$, we have

$$
\mathbf{G}_{\mathbf{a}}\left(A^{\prime} A^{\prime \prime}\right)=\sum_{\operatorname{Park}(u \otimes v)=\mathbf{a}} u \otimes v .
$$

For example, writing tensor products as biwords, one has

$$
\mathbf{G}_{4121}\left(A^{\prime} A^{\prime \prime}\right)=\sum_{a, b, c, d}\left[\begin{array}{cccc}
b & a & a & a \\
d & c & c+1 & c
\end{array}\right]
$$

with $b>a$, or $b=a$ and $d \geq c+3$.

Theorem 3.17 ([28]). The formula $\delta\left(\mathbf{G}_{\mathbf{a}}\right)=\mathbf{G}_{\mathbf{a}}\left(A^{\prime} A^{\prime \prime}\right)$ defines a coassociative coproduct on each homogeneous component $\mathbf{P Q S y m}_{n}^{*}$. Actually,

$$
\delta\left(\mathbf{G}_{\mathbf{a}}\right)=\sum_{\operatorname{Park}\left(\mathbf{a}^{\prime} \otimes \mathbf{a}^{\prime \prime}\right)=\mathbf{a}} \mathbf{G}_{\mathbf{a}^{\prime}} \otimes \mathbf{G}_{\mathbf{a}^{\prime \prime}},
$$

where $\mathbf{a}^{\prime}$ and $\mathbf{a}^{\prime \prime}$ are parking functions. By duality, the formula

$$
\mathbf{F}_{\mathbf{a}^{\prime}} * \mathbf{F}_{\mathbf{a}^{\prime \prime}}=\mathbf{F}_{\operatorname{Park}\left(\mathbf{a}^{\prime} \otimes \mathbf{a}^{\prime \prime}\right)}
$$

defines an associative product on each $\mathbf{P Q S y m} \mathbf{m}_{n}$.

Since $A$ is infinite, $\delta$ is compatible with the product of PQSym*.

EXAMPLE 3.18 .

(131) $\quad \delta \mathbf{G}_{4121}$

$$
\begin{aligned}
= & \left(\mathbf{G}_{2111}+\mathbf{G}_{3111}+\mathbf{G}_{4111}\right) \otimes\left(\mathbf{G}_{1232}+\mathbf{G}_{1121}+\mathbf{G}_{2121}+\mathbf{G}_{3121}+\mathbf{G}_{4121}\right) \\
& +\mathbf{G}_{1111} \otimes \mathbf{G}_{4121} .
\end{aligned}
$$

EXAMPLE 3.19 .

$$
\mathbf{F}_{211} * \mathbf{F}_{211}=\mathbf{F}_{311}, \quad \mathbf{F}_{211} * \mathbf{F}_{112}=\mathbf{F}_{312},
$$




$$
\begin{gathered}
\mathbf{F}_{211} * \mathbf{F}_{121}=\mathbf{F}_{321}, \quad \mathbf{F}_{112} * \mathbf{F}_{312}=\mathbf{F}_{213}, \\
\mathbf{F}_{31143231} * \mathbf{F}_{23571713}=\mathbf{F}_{61385451} .
\end{gathered}
$$

Note that although parking functions can be interpreted as chambers of the Shi arrangement, our internal product is not induced by the face semigroup of this arrangement. Indeed, one should obtain in particular an idempotent semigroup, which is clearly not the case.

The main tool for handling internal products of noncommutative symmetric functions is the splitting formula (see [7, Proposition 5.2]). It does not hold in PQSym, but one can find subalgebras of PQSym larger than Sym in which it remains true.

3.11. Subalgebras of PQSym. In the following sections, we present different subalgebras of PQSym, summarized in Figure 2 with the names of their natural bases:

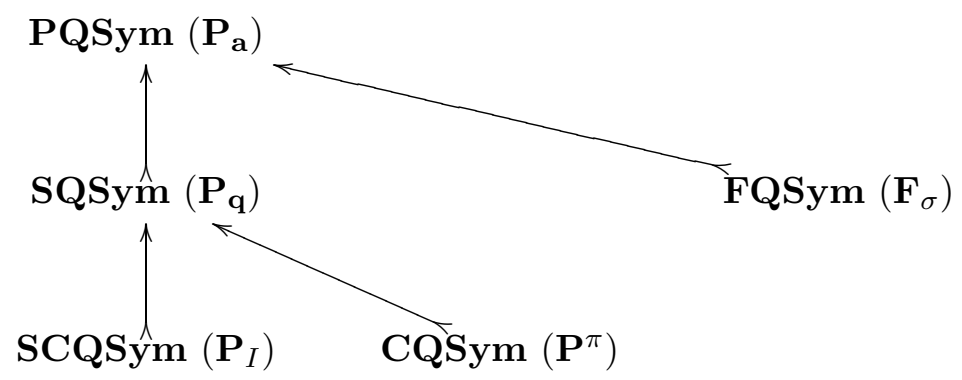

Fig. 2. Some subalgebras of PQSym

Here $\mathbf{a}$ is a parking function, $\sigma$ is a permutation, $\mathbf{q}$ is a parking quasiribbon, $I$ is a composition, and $\pi$ is a nondecreasing parking function.

\section{THE SCHRÖDER QUASI-SYMMETRIC HOPF ALGEBRA SQSym}

In Section 2.4, we recalled that the little Schröder numbers build up the Hilbert series of the free dendriform trialgebra on one generator, $\mathfrak{T} \mathfrak{D}$. We show in [29] that $\mathfrak{T D}$ realized on words has a natural structure of bidendriform bialgebra. In particular, this proves that there is a natural self-dual Hopf structure on $\mathfrak{T D}$.

But parking functions provide another way to find little Schröder numbers. Indeed, the number of classes of parking functions of length $n$ under the hypoplactic congruence is also equal to $s_{n}$. This construction leads to a non-self-dual Hopf algebra, denoted by SQSym. 
4.1. Hypoplactic classes of parking functions. Let $\equiv$ denote the hypoplactic congruence (see $[17,26])$. Recall that the equivalence classes of words under this congruence are parametrized by quasi-ribbon tableaux. A quasi-ribbon tableau of shape $I$ is a ribbon diagram $r$ of shape $I$ filled by letters in such a way that each row of $r$ is nondecreasing from left to right, and each column of $r$ is strictly increasing from top to bottom. A word is said to be a quasi-ribbon word of shape $I$ if it can be obtained by reading from bottom to top and from left to right the columns of a quasi-ribbon diagram of shape $I$. For example, the word 11425477 is a quasi-ribbon word since it is the reading of the following quasi-ribbon:

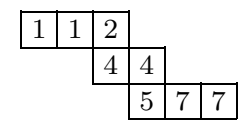

The hypoplactic classes of parking functions correspond to parking quasiribbons, that is, quasi-ribbon words that are parking functions. We denote this set by $\mathrm{PQR}$, and $\mathrm{PQR}_{n}$ is the set of quasi-ribbon parking functions of length $n$.

We will make use of a simple parametrization of the elements of PQR: define a segmented word as a finite sequence of nonempty words, separated by vertical bars, e.g., $232|14| 5 \mid 746$.

The parking quasi-ribbons can be represented as segmented nondecreasing parking functions where the bars only occur at positions $\cdots a \mid b \cdots$, with $a<b$. For example, the quasi-ribbon of equation (135) is represented by the word $112|44| 577$.

Clearly, a nondecreasing word containing exactly $l$ different letters admits $2^{l-1}$ segmentations.

On the other hand, the statistic $l$ (the length of the packed evaluation vector) on nondecreasing parking functions has the same distribution as the number of blocks in noncrossing partitions through the natural bijection. This is given by a classical $q$-Catalan number, $c_{n}(q)$ (see, e.g., [25]) and finally, the number of canonical packed words of length $n$ is $c_{n}(2)$, which is known to be equal to the Schröder number $s_{n}$.

For example, $c_{1}(q)=1, c_{2}(q)=1+q$ and $c_{3}(q)=1+3 q+q^{2}$, so that $c_{1}(2)=1, c_{2}(2)=3$ and $c_{3}(2)=11$ as one can check from (136) and (137). The coefficients of $c_{n}(q)$ are known as the Narayana numbers (sequence A001263 of Sloane's database [34]).

Here is the list of canonical hypoplactic parking functions for $n \geq 3$ :

$$
\{1\}, \quad\{11,12,1 \mid 2\},
$$

$$
\{111,112,11|2,113,11| 3,122,1|22,123,1| 23,12|3,1| 2 \mid 3\} .
$$

In the following, we will identify parking quasi-ribbons and their encodings as segmented words. 
4.2. The Schröder quasi-symmetric Hopf algebra SQSym. Let us denote by $\mathrm{P}(w)$ the hypoplactic $P$-symbol of a word $w$ (its quasi-ribbon). The P-symbols of parking functions are therefore parking quasi-ribbons. With a parking quasi-ribbon $\mathbf{q}$, we associate the elements

$$
\mathbf{P}_{\mathbf{q}}:=\sum_{\mathrm{P}(\mathbf{a})=\mathbf{q}} \mathbf{F}_{\mathbf{a}} \text { and } \mathbf{Q}_{\mathbf{q}}:=\overline{\mathbf{G}}_{\mathbf{a}}
$$

where $\bar{w}$ denotes the hypoplactic class of $w$. For example,

$$
\begin{gathered}
\mathbf{P}_{11 \mid 3}=\mathbf{F}_{131}+\mathbf{F}_{311}, \quad \mathbf{P}_{113}=\mathbf{F}_{113}, \\
\mathbf{Q}_{11 \mid 3}=\overline{\mathbf{G}}_{131}=\overline{\mathbf{G}}_{311}, \quad \mathbf{Q}_{113}=\overline{\mathbf{G}}_{113}, \\
\mathbf{Q}_{12 \mid 34}=\overline{\mathbf{G}}_{1324}=\overline{\mathbf{G}}_{3124}=\overline{\mathbf{G}}_{1342}=\overline{\mathbf{G}}_{3142}=\overline{\mathbf{G}}_{3412} .
\end{gathered}
$$

Theorem 4.1. The $\mathbf{P}_{\mathbf{q}}$ form a basis of a Hopf subalgebra of PQSym, denoted by SQSym. Its dual SQSym* is the quotient $\mathbf{P Q S y m} \mathbf{S O}^{*} / \mathcal{J}$ where $\mathcal{J}$ is the two-sided ideal generated by

$$
\left\{\mathbf{G}_{\mathbf{a}}-\mathbf{G}_{\mathbf{a}^{\prime}} \mid \mathbf{a} \equiv \mathbf{a}^{\prime}\right\} .
$$

Moreover, $\mathbf{G}_{\mathbf{a}} \equiv \mathbf{G}_{\mathbf{a}^{\prime}}$ iff $\mathbf{a} \equiv \mathbf{a}^{\prime}$, so that $\mathbf{S Q S y m} \mathbf{m}^{*} \simeq \mathbf{P Q S y m}^{*} / \equiv$. The dual basis of $\left(\mathbf{P}_{\mathbf{q}}\right)$ is then $\left(\mathbf{Q}_{\mathbf{q}}\right)$.

The dimension of the component of degree $n$ of SQSym and SQSym* is the little Schröder number (or super-Catalan number) $s_{n}$.

Proof. Let us begin with the elements $\overline{\mathbf{G}}_{\mathbf{a}}$. Since the hypoplactic equivalence is a congruence:

$$
u \equiv u^{\prime} \text { and } v \equiv v^{\prime} \Rightarrow u v \equiv u^{\prime} v^{\prime}
$$

these elements build up an algebra that we will denote by SQSym*. Since the hypoplactic congruence is compatible with the restriction to intervals, one easily checks that the coproduct of $\mathbf{G}_{\mathbf{a}}$ is compatible with the hypoplactic congruence, so that SQSym* is a Hopf algebra.

Recall that two words $u$ and $v$ are hypoplactically equivalent iff they have the same evaluation and $\operatorname{Std}(u)$ and $\operatorname{Std}(v)$ are hypoplactically equivalent. Since two words of the same evaluation have parkized words of the same evaluation as well, the same result applies if one replaces standardization by parkization: two words $u$ and $v$ of the same evaluation are hypoplactically equivalent iff their parkized words are. This proves that

$$
\mathbf{a} \equiv \mathbf{a}^{\prime} \Leftrightarrow \overline{\mathbf{G}}_{\mathbf{a}}=\overline{\mathbf{G}}_{\mathbf{a}^{\prime}} .
$$

So SQSym* is isomorphic to PQSym*/三 as a Hopf algebra.

Since the dual basis of $\mathbf{G}_{\mathbf{a}}$ in $\mathbf{P Q S y m}$ is $\mathbf{F}_{\mathbf{a}}$, one can write the duality bracket as

$$
\left\langle\mathbf{G}_{\mathbf{a}}, \mathbf{F}_{\mathbf{a}^{\prime}}\right\rangle=\delta_{\mathbf{a}, \mathbf{a}^{\prime}},
$$


where $\delta$ is the Kronecker symbol. Then the dual basis of $\mathbf{Q}_{q}=\overline{\mathbf{G}}_{\mathbf{a}}$ inherited from the dual Hopf algebras PQSym, PQSym* is naturally $\sum_{\mathbf{a}^{\prime} \equiv \mathbf{a}} \mathbf{F}_{\mathbf{a}^{\prime}}$, that is, $\mathbf{P}_{\mathbf{q}}$. It then follows without proof that the $\mathbf{P}_{\mathbf{q}}$ form a basis of a Hopf subalgebra of PQSym we will denote by SQSym, as it is the dual of SQSym*.

The dimensions are given by the little Schröder numbers since these numbers count the hypoplactic classes of parking functions.

Theorem 4.2. The product and coproduct rules for the $\mathbf{Q}_{\mathbf{q}}$ and $\mathbf{P}_{\mathbf{q}}$ are:

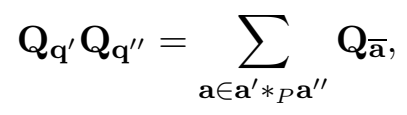

where $\mathbf{a}^{\prime}$ (resp. $\left.\mathbf{a}^{\prime \prime}\right)$ is in the hypoplactic class of $\mathbf{q}^{\prime}$ (resp. $\left.\mathbf{q}^{\prime \prime}\right)$;

$$
\begin{gathered}
\Delta \mathbf{Q}_{\mathbf{q}}=\sum_{\substack{u, v ; \mathbf{q}=u \mid v[|u|] \text { or } \mathbf{q}=u \cdot v[|u|] \\
\mathbf{P}_{\mathbf{q}^{\prime}} \mathbf{P}_{\mathbf{q}^{\prime \prime}}=\mathbf{P}_{\mathbf{q}^{\prime} \mid \mathbf{r}^{\prime \prime}}+\mathbf{P}_{\mathbf{q}^{\prime} \mathbf{r}^{\prime \prime}}}} \mathbf{G}_{u} \otimes \mathbf{G}_{v},
\end{gathered}
$$

where $\mathbf{r}^{\prime \prime}=\mathbf{q}^{\prime \prime}\left[\left|\mathbf{q}^{\prime}\right|\right]$; and

$$
\Delta \mathbf{P}_{\mathbf{q}}=\sum_{\mathbf{q}^{\prime}, \mathbf{q}^{\prime \prime}} \mathbf{P}_{\mathbf{q}^{\prime}} \otimes \mathbf{P}_{\mathbf{q}^{\prime \prime}}
$$

where the sum is taken over the hypoplactic classes $\mathbf{q}^{\prime}$ and $\mathbf{q}^{\prime \prime}$ such that their canonical elements $c^{\prime}$ and $c^{\prime \prime}$ can be obtained as parkized words of the prefix and the suffix of an element of the hypoplactic class $\mathbf{q}$.

Proof. The formulas for the product and coproduct of the $\mathbf{Q}$ come from the formulas of the $\mathbf{G}$ in $\mathbf{P Q S y m}$. The formulas for the $\mathbf{P}$ are then easily derived from the previous ones by duality.

For example,

$$
\begin{aligned}
& \mathbf{Q}_{1 \mid 2} \mathbf{Q}_{1}=\mathbf{Q}_{1 \mid 23}+\mathbf{Q}_{1 \mid 22}+\mathbf{Q}_{12 \mid 3}+\mathbf{Q}_{11 \mid 3}+\mathbf{Q}_{11 \mid 2}+\mathbf{Q}_{1|2| 3}, \\
& \Delta \mathbf{Q}_{11|34| 55}=1 \otimes \mathbf{Q}_{11|34| 55}+\mathbf{Q}_{11} \otimes \mathbf{Q}_{12 \mid 33}+\mathbf{Q}_{11 \mid 3} \otimes \mathbf{Q}_{1 \mid 22} \\
& +\mathbf{Q}_{11 \mid 34} \otimes \mathbf{Q}_{11}+\mathbf{Q}_{11|34| 55} \otimes 1, \\
& \Delta \mathbf{P}_{11 \mid 3}=1 \otimes \mathbf{P}_{11 \mid 3}+\mathbf{P}_{1} \otimes\left(\mathbf{P}_{1 \mid 2}+\mathbf{P}_{11}\right)+\left(\mathbf{P}_{21}+\mathbf{P}_{12}\right) \otimes \mathbf{P}_{1}+\mathbf{P}_{11 \mid 3} \otimes 1 .
\end{aligned}
$$

4.3. SQSym is not self-dual. Some simple computations prove that SQSym and SQSym* are not isomorphic Hopf algebras since the primitive Lie algebra of SQSym* is of dimension 6 in degree 3, spanned by

$$
\begin{aligned}
& \mathbf{Q}_{111}, \mathbf{Q}_{112}, \mathbf{Q}_{11 \mid 2}, \mathbf{Q}_{122}-\mathbf{Q}_{1 \mid 22}, \mathbf{Q}_{113}-\mathbf{Q}_{11 \mid 3}, \\
& \mathbf{Q}_{123}-\mathbf{Q}_{1 \mid 23}-\mathbf{Q}_{12 \mid 3}+\mathbf{Q}_{1|2| 3},
\end{aligned}
$$

whereas it is of dimension 7 in SQSym, spanned by 


$$
\begin{array}{ll}
\mathbf{P}_{123}-\mathbf{P}_{112}-\mathbf{P}_{122}+\mathbf{P}_{111}, & \mathbf{P}_{1 \mid 22}-\mathbf{P}_{11 \mid 2}-\mathbf{P}_{112}+\mathbf{P}_{122} \\
\mathbf{P}_{1 \mid 23}-\mathbf{P}_{11 \mid 2}-\mathbf{P}_{112}+\mathbf{P}_{111}, & \mathbf{P}_{12 \mid 3}-\mathbf{P}_{11 \mid 2}-\mathbf{P}_{112}+\mathbf{P}_{111}, \\
\mathbf{P}_{113}-\mathbf{P}_{112}, \quad \mathbf{P}_{11 \mid 3}-\mathbf{P}_{11 \mid 2}, & \mathbf{P}_{1|2| 3}-\mathbf{P}_{11 \mid 2}+\mathbf{P}_{1 \mid 22} .
\end{array}
$$

In particular, it is impossible to endow SQSym or SQSym* with a bidendriform bialgebra structure since both would then be self-dual. We cannot use the machinery of Foissy to investigate the freeness of both algebras and their primitive Lie algebras, but we can do it by hand.

4.4. Algebraic structure of SQSym* and SQSym. Since we know that the primitive Lie algebra of SQSym is of dimension seven in degree 3, SQSym* cannot be free and, indeed, one finds the relation

$$
\mathbf{Q}_{1}\left(\mathbf{Q}_{11}+\mathbf{Q}_{12}\right)=\left(\mathbf{Q}_{11}+\mathbf{Q}_{12}\right) \mathbf{Q}_{1}
$$

We now move to SQSym. Consider the set PQS of parking quasi-ribbons that cannot be obtained as a nontrivial shifted concatenation of parking quasi-ribbons. They are the parking quasi-ribbons having a bar whenever the underlying nondecreasing parking function has a breakpoint. For example, here are the elements of $\mathrm{PQS}_{n}$ for $n \leq 4$.

$$
\begin{aligned}
& \{1\}, \quad\{11,1 \mid 2\}, \quad\{111,112,11|2,11| 3,1|22,1| 2 \mid 3\} \\
& \{1111,1112,111|2,1113,111| 3,111|4,1122,11| 22, \\
& 1123,11|23,112| 3,11|2| 3,112|4,11| 2|4,11| 33, \\
& 11|3| 4,1|222,1| 223,1|22| 3,1|22| 4,1|2| 33,1|2| 3 \mid 4\} .
\end{aligned}
$$

Since the elements of PQS are those that never occur in a nontrivial shifted concatenation of elements of PQR, any element $\mathbf{q}$ of PQR decomposes uniquely as a shifted product $\mathbf{q}_{1} \bullet \cdots \bullet \mathbf{q}_{k}$ where all the $\mathbf{q}_{k}$ are in PQS. Define then

$$
\mathbf{P}^{\mathbf{q}}=\mathbf{P}_{\mathbf{q}_{1}} \cdots \mathbf{P}_{\mathbf{q}_{k}}
$$

Proposition 4.3. The $\mathbf{P}^{\mathbf{q}}$ form a multiplicative basis of SQSym. In particular, SQSym is free as an algebra.

Proof. The $\mathbf{P}^{\mathbf{q}}$ generate the same algebra as the $\mathbf{P}_{\mathbf{q}}$ since they are triangular over the $\mathbf{P}_{\mathbf{q}}$ : each term $\mathbf{P}^{\mathbf{q}}$ begins with $\mathbf{P}_{\mathbf{q}}$ followed by elements of $\mathrm{PQR}$ that are shifted concatenations of strictly lower elements of PQS.

Since SQSym is free, one can compute the generating series of its generating set. Recall that the generating series of $s_{n}$ is $S(t):=\frac{1+t-\sqrt{1-6 t+t^{2}}}{4 t}$, so that

$$
U(t):=1-1 / S(t)=\frac{1-t-\sqrt{1-6 t+t^{2}}}{2},
$$

that is, the generating series of large Schröder numbers $s_{n}^{\prime}$ (A006318 in [34]), obviously equal to $2 s_{n}$ thanks to the previous formula. So 
Proposition 4.4. The sets $\mathrm{PQS}_{n}$ are enumerated by the large Schröder numbers.

Proof. Even if the algebraic construction has already proved this result, we provide a bijective proof in order to enlighten the relation between the large and the little Schröder numbers from the point of view of parking functions.

We split $\mathrm{PQS}_{n}$ in two and provide a bijection between both sets and $\mathrm{PQR}_{n-1}$, the set of parking quasi-ribbons of length $n-1$.

Let $\mathrm{PQS}_{n}^{\prime}$ be the subset of $\mathrm{PQS}_{n}$ consisting of the elements whose underlying parking function is prime. The bijection between $\mathrm{PQS}_{n}^{\prime}$ and $\mathrm{PQR}_{n-1}$ is trivial: it consists in adding or removing 1 at the beginning of the parking function.

Let $\mathrm{PQS}_{n}^{\prime \prime}$ be the complementary subset of $\mathrm{PQS}_{n}$. The bijection is the following: start from an element of $\mathrm{PQR}_{n-1}$. If it belongs to $\mathrm{PQS}_{n-1}$, then add a bar and $n$ to its end. Otherwise, let $i$ be the smallest integer greater than 1 such that $i-1$ is a breakpoint and there is no bar before the first $i$. Then insert a bar and an $i$ before the first $i$. This element satisfies the requirements of $\mathrm{PQS}_{n}$ since it can have breakpoints only to the left of $i$ and, by hypothesis, all those breakpoints are followed by a bar. Moreover, this element has a breakpoint, so belongs to $\mathrm{PQS}_{n}^{\prime \prime}$. For example, the image of $11|2| 455 \mid 669$ is $11|2| 4|55| 669$, since there is a breakpoint at 4 with no bar before the first 5 .

The reverse bijection consists in considering the rightmost breakpoint $i$ of the underlying parking function of an element of $\mathrm{PQS}_{n}^{\prime \prime}$ and remove $i+1$ with the bar before it. The result belongs to $\mathrm{PQR}_{n-1}$ since we removed the letter just after the rightmost breakpoint.

Finally, it is a bijection between $\mathrm{PQS}_{n}^{\prime \prime}$ and $\mathrm{PQR}_{n-1}$ since the operations are inverse to each other and the image of each set is included in the other.

The next proposition summarizes the structures of the algebras SQSym and SQSym*.

Proposition 4.5. The algebra SQSym is a Hopf algebra of dimension $s_{n}$ in degree $n$. It is not self-dual since $\mathbf{S Q S y m}$ is free as an algebra whereas SQSym* is not.

4.5. SQSym* as a combinatorial Hopf algebra. The embedding of (93) induces an embedding

$$
\text { QSym } \simeq \text { FQSym }^{*} /\left(\mathcal{J} \cap \text { FQSym }^{*}\right) \rightarrow \mathbf{P Q S y m}^{*} / \mathcal{J}=\text { SQSym }^{*} .
$$

In particular, we see that SQSym* contains a large commutative subalgebra. 


\subsection{Primitive Lie algebras of SQSym and SQSym*. As SQSym*} contains QSym as a subalgebra, its primitive Lie algebra cannot be free and one easily finds

$$
\left[\mathbf{Q}_{1}, \mathbf{Q}_{12}-\mathbf{Q}_{1 \mid 2}+\mathbf{Q}_{11}\right]=0 \text {. }
$$

The first dimensions for the primitive Lie algebra of SQSym are 1, 2, 7, 25,102 , with no relations between those elements in those degrees so that one can conjecture that it is free as a Lie algebra.

4.7. Schröder ribbons. In the algebra Sym, the products of noncommutative complete functions split into sums of ribbon Schur functions, using a simple order on compositions. To get an analogous construction in our case, we define a partial order on segmented nondecreasing parking functions.

Let $\pi$ be a segmented nondecreasing parking function and $\operatorname{Ev}(\pi)$ be its segmented evaluation vector, that is, its evaluation vector with separators between the $i$ th and $(i+1)$ th element if $i$ and $i+1$ are separated by a bar in $\pi$. The successors of $\pi$ are the segmented nondecreasing parking functions whose evaluations are given by the following algorithm: given two nonzero elements of $\operatorname{Ev}(\pi)$ not separated by a bar with only zeros between them, replace the left one by the sum of both and the right one by 0 .

For example, the successors of 11|3346 are 11|3336 and 11|3344.

By transitive closure, the successor map gives rise to a partial order $\succeq$ on segmented nondecreasing parking functions.

Now, define the Schröder ribbons by

$$
\mathbf{P}_{\mathbf{q}}=: \sum_{\mathbf{q}^{\prime} \succeq \mathbf{q}} \mathbf{R}_{\mathbf{q}^{\prime}}
$$

or, by Möbius inversion on the boolean lattice,

$$
\mathbf{R}_{\mathbf{q}}:=\sum_{\mathbf{q}^{\prime} \succeq \mathbf{q}}(-1)^{f\left(\mathbf{q}, \mathbf{q}^{\prime}\right)} \mathbf{P}_{\mathbf{q}^{\prime}}
$$

where $f(u, v)$ is the difference between the numbers of different letters in $u$ and in $v$.

For example,

$$
\begin{aligned}
\mathbf{P}_{11 \mid 34} & =\mathbf{R}_{11 \mid 34}+\mathbf{R}_{11 \mid 33}, \\
\mathbf{P}_{11 \mid 3346} & =\mathbf{R}_{11 \mid 3346}+\mathbf{R}_{11 \mid 3336}+\mathbf{R}_{11 \mid 3344}+\mathbf{R}_{11 \mid 3333}, \\
\mathbf{R}_{11 \mid 3346} & =\mathbf{P}_{11 \mid 3346}-\mathbf{P}_{11 \mid 3336}-\mathbf{P}_{11 \mid 3344}+\mathbf{P}_{11 \mid 3333 .} .
\end{aligned}
$$

Proposition 4.6. The product of two ribbons is given by

$$
\mathbf{R}_{\mathbf{q}^{\prime}} \mathbf{R}_{\mathbf{q}^{\prime \prime}}=\mathbf{R}_{\mathbf{q}^{\prime} \mid \mathbf{r}^{\prime \prime}}+\mathbf{R}_{\mathbf{q}^{\prime} \mathbf{r}^{\prime \prime}}+\mathbf{R}_{\mathbf{q}^{\prime} \triangleright \mathbf{r}^{\prime \prime}}
$$

where $\mathbf{r}^{\prime \prime}=\mathbf{q}^{\prime \prime}\left[\left|\mathbf{q}^{\prime}\right|\right]$ and $\mathbf{q}^{\prime} \triangleright \mathbf{r}^{\prime \prime}$ is the successor of $\mathbf{q}^{\prime} \mathbf{r}^{\prime \prime}$ obtained by decreasing the smallest letters of $\mathbf{r}^{\prime \prime}$ down to the value of the greatest letters of $\mathbf{q}^{\prime}$. 
Proof. Let $p$ be the length of $\mathbf{q}^{\prime}$. Let us expand $\mathbf{R}_{\mathbf{q}^{\prime}} \mathbf{R}_{\mathbf{q}^{\prime \prime}}$ in the $\mathbf{P}$ basis. One gets an alternating sum of $\mathbf{P}$ indexed by the successors of $\mathbf{q}^{\prime} \mathbf{r}^{\prime \prime}$ having different $p$ th and $(p+1)$ st letters or indexed by the successors of $\mathbf{q}^{\prime} \mid \mathbf{r}^{\prime}$. The second set obviously sums up to $\mathbf{R}_{\mathbf{q}^{\prime} \mid \mathbf{r}^{\prime \prime}}$. The first set is part of all successors of $\mathbf{q}^{\prime} \mathbf{r}^{\prime \prime}$, the missing set being all successors of $\mathbf{q}^{\prime} \triangleright \mathbf{r}^{\prime \prime}$.

As the sign of an element depends only on its number of different letters, the result follows.

For example,

$$
\begin{aligned}
\mathbf{R}_{1} \mathbf{R}_{1 \mid 2} & =\mathbf{R}_{1|2| 3}+\mathbf{R}_{12 \mid 3}+\mathbf{R}_{11 \mid 3}, \\
\mathbf{R}_{11 \mid 3} \mathbf{R}_{113} & =\mathbf{R}_{11|3| 446}+\mathbf{R}_{11 \mid 3446}+\mathbf{R}_{11 \mid 3336} .
\end{aligned}
$$

4.8. Dendriform structures on SQSym. Let us now consider the other structures that can be put on SQSym and SQSym*. First note that the product rules of PQSym* as a tridendriform algebra are compatible with the hypoplactic congruence, so that SQSym* is a tridendriform algebra. But it is not free since

$$
\mathbf{Q}_{11 \mid 2}=\overline{\mathbf{G}}_{212}=\overline{\mathbf{G}}_{221},
$$

which can be rewritten as

$$
\left(\mathbf{Q}_{1} \succ \mathbf{Q}_{1}\right) \circ \mathbf{Q}_{1}=\mathbf{Q}_{1} \circ\left(\mathbf{Q}_{1} \succ \mathbf{Q}_{1}\right),
$$

a relation that is not a consequence of the tridendriform relations.

We already mentioned that SQSym* cannot have a bidendriform bialgebra structure since it would imply that $\mathbf{S Q S y m} *$ is self-dual. In our realization of the bidendriform bialgebra PQSym, the explanation comes from the fact that the hypoplactic congruence is not compatible with the codendriform definitions since, for example,

$$
\Delta_{\ll} \mathbf{G}_{221}=\mathbf{G}_{1} \otimes \mathbf{G}_{11} \quad \text { whereas } \quad \Delta_{\ll} \mathbf{G}_{212}=0 .
$$

\section{THE CATALAN QUASI-SYMMETRIC HOPF ALGEBRA CQSym}

\subsection{The Hopf algebra CQSym}

5.1.1. Nondecreasing parking functions and noncrossing partitions. As already mentioned, nondecreasing parking functions form a Catalan set. There are dozens of possibilities to identify them to other combinatorial objects. However, parking functions are known to be related to noncrossing partitions (see $[3,36,37]$ ), and there is a simple bijection between nondecreasing parking functions and noncrossing partitions. Starting with a noncrossing partition, e.g.,

$$
\pi=13|2| 45,
$$


one replaces all the letters of each block by its minimum, and reorders them as a nondecreasing word:

$$
13|2| 45 \rightarrow 11244
$$

which is a parking function. In the following, we identify nondecreasing parking functions and noncrossing partitions via this bijection.

5.1.2. The Catalan Hopf algebra CQSym. For a general a $\in \mathrm{PF}_{n}$, let $\mathrm{NC}(\mathbf{a})$ be the noncrossing partition corresponding to $\mathbf{a}^{\uparrow}$ by the inverse bijection, e.g., $\mathrm{NC}(42141)=\pi$ as above.

Then define $\mathbf{P}^{\pi}$ as the sum of all permutations of the nondecreasing word corresponding to the given noncrossing partition:

$$
\mathbf{P}^{\pi}:=\sum_{\mathbf{a} ; \mathrm{NC}(\mathbf{a})=\pi} \mathbf{F}_{\mathbf{a}} .
$$

THEOREM 5.1. The elements $\mathbf{P}^{\pi}$, where $\pi$ runs over noncrossing partitions, span a cocommutative Hopf subalgebra of PQSym with product and coproduct given by

$$
\begin{gathered}
\mathbf{P}^{\pi}=\mathbf{P}_{u, v ;(u \cdot v)^{\dagger}=\pi}^{\pi^{\pi^{\prime \prime}}=} \mathbf{P}^{\pi^{\prime} \bullet \pi^{\prime \prime}}, \\
\mathbf{P}^{\operatorname{Park}(u)} \otimes \mathbf{P}^{\operatorname{Park}(v)},
\end{gathered}
$$

where $u$ and $v$ run over the set of nondecreasing words.

Moreover, as an algebra, it is isomorphic to the algebra of the free semigroup of noncrossing partitions under the operation of concatenation of diagrams.

Proof. Equation (176) follows from (47): indeed, any permutation of $\pi^{\prime} \bullet \pi^{\prime \prime}$ is uniquely obtained as the shifted shuffle of a permutation of $\pi^{\prime}$ with a permutation of $\pi^{\prime \prime}$. The converse is obvious.

Equation (177) comes from (52): consider the relation $P(p, q)$ on words which are pairs $\left(w, w^{\prime}\right)$ of words $w$ and $w^{\prime}$ of length $p+q$ such that the sorted words of the prefix of length $p$ (resp. suffix of length $q$ ) of $w$ and $w^{\prime}$ are equal. By definition of $\mathbf{P}^{\pi}$, it is a sum of such classes, so that $\Delta \mathbf{P}^{\pi}$ decomposes as a sum of tensor products of the form $\mathbf{P}^{\pi^{\prime}} \otimes \mathbf{P}^{\pi^{\prime \prime}}$. The sum on the right-hand side of (177) is exactly over representatives of the equivalence classes, hence the result. Formula (177) proves that the coalgebra CQSym is cocommutative.

Moreover, since CQSym is a subalgebra and a sub-coalgebra of PQSym, the product and the coproduct of CQSym are compatible, so that CQSym is endowed with a graded bialgebra structure, and therefore, with a Hopf algebra structure. 
This algebra will be called the Catalan subalgebra of PQSym and denoted by CQSym.

For example, one has

$$
\begin{gathered}
\mathbf{P}^{11} \mathbf{P}^{1233}=\mathbf{P}^{113455}, \quad \mathbf{P}^{1124} \mathbf{P}^{1223}=\mathbf{P}^{11245667} \\
\Delta \mathbf{P}^{1124}=1 \otimes \mathbf{P}^{1124}+\mathbf{P}^{1} \otimes\left(\mathbf{P}^{112}+\mathbf{P}^{113}+\mathbf{P}^{123}\right) \\
+\mathbf{P}^{11} \otimes \mathbf{P}^{12}+\mathbf{P}^{12} \otimes\left(\mathbf{P}^{11}+2 \mathbf{P}^{12}\right) \\
+\left(\mathbf{P}^{112}+\mathbf{P}^{113}+\mathbf{P}^{123}\right) \otimes \mathbf{P}^{1}+\mathbf{P}^{1124} \otimes 1 .
\end{gathered}
$$

Since the nondecreasing parking functions that never occur in a nontrivial shifted concatenation of such elements are the connected nondecreasing parking functions, any $\pi$ decomposes uniquely as a shifted product $\pi_{1} \bullet \cdots \bullet \pi_{k}$ where all the $\pi_{k}$ are connected.

Proposition 5.2. The $\mathbf{P}$ form a multiplicative basis of CQSym. In particular, CQSym is free as an algebra.

Here are the connected nondecreasing parking functions up to length 4: (180)

$$
\{1\}, \quad\{11\}, \quad\{111,112\}, \quad\{1111,1112,1113,1122,1123\} .
$$

Since CQSym is free, one can compute the generating series of its generating set. Recall that the generating series of $C_{n}$ is $C(t):=(1-\sqrt{1-4 t}) / 2 t$, so that

$$
C N(t):=1-1 / C(t)=t \frac{1-\sqrt{1-6 t+t^{2}}}{2},
$$

that is, the generating series of shifted Catalan numbers $C_{n-1}$. Indeed, the connected nondecreasing parking functions are obtained by concatenating a 1 to the left of all nondecreasing parking functions.

5.1.3. Algebraic structure of CQSym. Following Reutenauer [33, p. 58], denote by $\pi_{1}$ the Eulerian idempotent, i.e., the endomorphism of CQSym defined by $\pi_{1}=\log ^{*}(\mathrm{Id})$ where $\log ^{*}$ means that the logarithm is taken in the convolution algebra End ${ }^{\text {gr }}(\mathbf{C Q S y m})$ of graded endomorphisms. It is obvious, thanks to the definition of $\mathbf{P}^{\pi}$, that

$$
\pi_{1}\left(\mathbf{P}^{\pi}\right)=\mathbf{P}^{\pi}+\cdots,
$$

where the dots stand for terms $\mathbf{P}^{\gamma}$ where $\gamma$ is not connected. So the family $\pi_{1}\left(\mathbf{P}^{\alpha}\right)$ where $\alpha$ runs over all connected nondecreasing parking functions is a free set of primitive generators of CQSym. In particular, they generate a free Lie algebra (see, e.g., [13] for more details) whose Hilbert series is given by

$$
t+t^{2}+3 t^{3}+8 t^{4}+25 t^{5}+75 t^{6}+245 t^{7}+800 t^{8}+O\left(t^{9}\right) .
$$

The sequence is referenced in Sloane's database as A022553 [34]. It counts Lyndon words $l$ of even length $2 n$ with an equal number of $a$ and $b$. So 
the free Lie algebra of primitive elements of CQSym is isomorphic to the Lie subalgebra $\mathfrak{L}$ of the free Lie algebra $\operatorname{Lie}(a, b)$ consisting of the elements with an equal number of $a$ and $b$. One can then prove that the standard bracketings of the Lyndon words $l$ with the same number of $a$ and $b$ such that $l=l^{\prime} \cdot b$ with $l^{\prime}$ also being a Lyndon word generate a free Lie algebra. Since those particular Lyndon words are enumerated by the shifted Catalan numbers, one can conclude that they generate $\mathfrak{L}$.

5.2. The dual Hopf algebra CQSym*. Let us denote by $\mathcal{M}_{\pi}$ the dual basis of $\mathbf{P}^{\pi}$ in the commutative algebra CQSym*. Since CQSym is the subalgebra of PQSym obtained by summing all permutations of nondecreasing parking functions, CQSym* is the quotient of PQSym* by the relations $\mathbf{G}_{\mathbf{a}} \equiv \mathbf{G}_{\mathbf{b}}$ if $\mathbf{a}^{\uparrow}=\mathbf{b}^{\uparrow}$.

It is then immediate (see (61)) that the multiplication in this basis is given by

$$
\mathcal{M}_{\pi^{\prime}} \mathcal{M}_{\pi^{\prime \prime}}=\sum_{\mathbf{a} \in \pi^{\prime} * \pi^{\prime \prime}} \mathcal{M}_{\mathbf{a}^{\uparrow}}
$$

For example,

$$
\begin{aligned}
\mathcal{M}_{1} \mathcal{M}_{12}= & \mathcal{M}_{112}+\mathcal{M}_{113}+\mathcal{M}_{122}+3 \mathcal{M}_{123} \\
\mathcal{M}_{12} \mathcal{M}_{11}= & \mathcal{M}_{1112}+\mathcal{M}_{1113}+\mathcal{M}_{1114}+\mathcal{M}_{1123}+\mathcal{M}_{1124} \\
& +\mathcal{M}_{1134}+\mathcal{M}_{1222}+\mathcal{M}_{1223}+\mathcal{M}_{1224}+\mathcal{M}_{1233}
\end{aligned}
$$

TheOREM 5.3. The algebra CQSym* is embedded in the polynomial algebra $\mathbb{C}\left[x_{1}, x_{2}, \ldots\right]$ by

$$
\mathcal{M}_{\pi}=\sum_{\operatorname{Park}(w)=\pi} \underline{w}
$$

where $\underline{w}$ is the commutative image of $w\left(\right.$ i.e., $\left.a_{i} \mapsto x_{i}\right)$.

Proof. The result follows from (104) and from the fact that quotienting PQSym* by the relations $\mathbf{G}_{\mathbf{a}} \equiv \mathbf{G}_{\mathbf{b}}$ if $\mathbf{a}^{\uparrow}=\mathbf{b}^{\uparrow}$ amounts to taking the commutative image of words, transforming these into monomials.

For example,

$$
\begin{aligned}
& \mathcal{M}_{111}=\sum_{i} x_{i}^{3}, \\
& \mathcal{M}_{112}=\sum_{i} x_{i}^{2} x_{i+1}, \\
& \mathcal{M}_{113}=\sum_{i, j ; j \geq i+2} x_{i}^{2} x_{j},
\end{aligned}
$$




$$
\begin{aligned}
\mathcal{M}_{122} & =\sum_{i, j ; i<j} x_{i} x_{j}^{2}, \\
\mathcal{M}_{123} & =\sum_{i, j, k ; i<j<k} x_{i} x_{j} x_{k} .
\end{aligned}
$$

The packed evaluation vector $t(w)$ of $w$ is obtained from $\operatorname{Ev}(w)$ by removing all its zeros. For example, for $w=3117291781329, \operatorname{Ev}(w)=$ $(4,2,2,0,0,0,2,1,2)$ and $t(w)=(4,2,2,2,1,2)$.

We can now see that CQSym* contains QSym as a subalgebra. The embedding of QSym into CQSym* is given by

$$
\gamma\left(M_{I}\right):=\sum_{t(\pi)=I} \mathcal{M}_{\pi}
$$

For example,

$$
\begin{aligned}
& M_{3}=\mathcal{M}_{111}, \quad M_{21}=\mathcal{M}_{112}+\mathcal{M}_{113}, \\
& M_{12}=\mathcal{M}_{122}, \quad M_{111}=\mathcal{M}_{123} .
\end{aligned}
$$

5.3. Catalan ribbons. As already done for the Schröder algebras, we define a partial order on nondecreasing parking functions.

Let $\pi$ be a nondecreasing parking function and $\operatorname{Ev}(\pi)$ be its evaluation vector. The successors of $\pi$ are the nondecreasing parking functions whose evaluations are given by the following algorithm: given two nonzero elements of $\operatorname{Ev}(\pi)$ with only zeros between them, replace the left one by the sum of both and the right one by 0 .

For example, the successors of 113346 are 111146, 113336, and 113344.

By transitive closure, the successor map gives rise to a partial order on nondecreasing parking functions. We will write $\pi^{\prime} \succeq \pi$ if $\pi^{\prime}$ is obtained from $\pi$ by successive applications of successor maps.

Now, define the Catalan ribbon functions by

$$
\mathbf{P}^{\pi}=: \sum_{\pi^{\prime} \succeq \pi} \mathbf{R}_{\pi^{\prime}}
$$

This last equation completely defines the $\mathbf{R}_{\pi}$.

For example,

$$
\begin{aligned}
\mathbf{P}^{113346}= & \mathbf{R}_{113346}+\mathbf{R}_{113344}+\mathbf{R}_{113336}+\mathbf{R}_{113333} \\
& +\mathbf{R}_{111146}+\mathbf{R}_{111144}+\mathbf{R}_{111116}+\mathbf{R}_{111111} \\
\mathbf{R}_{113346}= & \mathbf{P}^{113346}-\mathbf{P}^{113344}-\mathbf{P}^{113336}+\mathbf{P}^{113333} \\
& -\mathbf{P}^{111146}+\mathbf{P}^{111144}+\mathbf{P}^{111116}-\mathbf{P}^{111111}
\end{aligned}
$$

Note that, by Möbius inversion of the boolean lattice, the coefficient of $\mathbf{P}^{\pi^{\prime}}$ 
in $\mathbf{R}^{\pi}$ is -1 to the number of different letters in $\pi$ minus the number of different letters in $\pi^{\prime}$.

This definition is compatible with the definition of commutative ribbon Schur functions since if one considers the morphism

$$
\phi: \mathbf{C Q S y m} \rightarrow \text { Sym }, \quad \mathbf{P}^{\pi} \mapsto S^{c(\pi)},
$$

then the image $\phi\left(\mathbf{R}_{\pi}\right)$ is equal to $R_{c(\pi)}$.

Proposition 5.4. The product of two $\mathbf{R}$ functions is

$$
\mathbf{R}_{\pi^{\prime}} \mathbf{R}_{\pi^{\prime \prime}}=\mathbf{R}_{\pi^{\prime} \bullet \pi^{\prime \prime}}+\mathbf{R}_{\pi^{\prime} \triangleright \pi^{\prime \prime}}
$$

where $\pi^{\prime} \triangleright \pi^{\prime \prime}$ is the successor of $\pi^{\prime} \bullet \pi^{\prime \prime}$ obtained by decreasing the smallest letters of $\pi^{\prime \prime}$ down to the greatest letters of $\pi^{\prime}$.

Proof. Let $p$ be the length of $\pi^{\prime}$. Let us expand $\mathbf{R}_{\pi^{\prime} \bullet \pi^{\prime \prime}}$ in the $\mathbf{P}$ basis. One gets the alternating sum of $\mathbf{P}$ indexed by successors of $\pi^{\prime} \bullet \pi^{\prime \prime}$. Those successors split into two disjoint subsets: the successors having the $p$ th and $(p+1)$ th letters equal and the others. The first set corresponds to the successors of $\pi^{\prime} \triangleright \pi^{\prime \prime}$ whereas the second set corresponds to the $w^{\prime} \bullet w^{\prime \prime}$ where $w^{\prime} \succeq \pi^{\prime}$ and $w^{\prime \prime} \succeq \pi^{\prime \prime}$.

As the sign of an element depends only on its number of different letters, the alternating sum of the first set gives $-\mathbf{R}_{\pi^{\prime} \triangleright \pi^{\prime \prime}}$, whereas the sum of the second set yields $\mathbf{R}_{\pi^{\prime}} \mathbf{R}_{\pi^{\prime \prime}}$.

For example,

$$
\begin{aligned}
& \mathbf{R}_{11224} \mathbf{R}_{113}=\mathbf{R}_{11224668}+\mathbf{R}_{11224448} \\
& \mathbf{R}_{113} \mathbf{R}_{11224}=\mathbf{R}_{11344557}+\mathbf{R}_{11333557}
\end{aligned}
$$

5.4. Internal product. Define the parkized word of a bimonomial as the nondecreasing parking function obtained by parkizing its lexicographically sorted biword. Recall that bimonomials can be encoded as matrices, the entry $A_{i j}$ being the number of bi-letters $(i j)$ in the biword, so that it makes sense to speak of the parkized word of a matrix.

Theorem 5.5 ([28]). The homogeneous components $\mathbf{C Q S y m}_{n}$ of the Catalan algebra are stable under the internal product $*$. More precisely,

$$
\mathbf{P}^{\pi^{\prime}} * \mathbf{P}^{\pi^{\prime \prime}}=\sum_{\pi} \mathbf{P}^{\pi}
$$

where $\pi$ runs over the parkized words of all nonnegative integer matrices with row sum $\operatorname{Ev}\left(\pi^{\prime}\right)$ and column sum $\operatorname{Ev}\left(\pi^{\prime \prime}\right)$. 
EXAMPLE 5.6.

$$
\begin{gathered}
\mathbf{P}^{1123} * \mathbf{P}^{1111}=\mathbf{P}^{1134}, \quad \mathbf{P}^{1111} * \mathbf{P}^{1123}=\mathbf{P}^{1123} \\
\mathbf{P}^{1123} * \mathbf{P}^{1112}=2 \mathbf{P}^{1134}+\mathbf{P}^{1234} \\
\mathbf{P}^{1122} * \mathbf{P}^{1224}=\mathbf{P}^{1134}+\mathbf{P}^{1233}+2 \mathbf{P}^{1234} \\
\mathbf{P}^{1123} * \mathbf{P}^{1224}=2 \mathbf{P}^{1134}+5 \mathbf{P}^{1234}
\end{gathered}
$$

The matrices appearing in the last product are

$$
\begin{aligned}
& \left(\begin{array}{cccc}
1 & 1 & \cdot & \cdot \\
\cdot & 1 & \cdot & \cdot \\
\cdot & \cdot & \cdot & 1
\end{array}\right),\left(\begin{array}{cccc}
1 & 1 & \cdot & \cdot \\
\cdot & \cdot & \cdot & 1 \\
\cdot & 1 & \cdot & .
\end{array}\right),\left(\begin{array}{cccc}
1 & \cdot & \cdot & 1 \\
\cdot & 1 & \cdot & \cdot \\
\cdot & 1 & \cdot & \cdot
\end{array}\right),\left(\begin{array}{cccc}
\cdot & 2 & \cdot & \cdot \\
1 & \cdot & \cdot & \cdot \\
\cdot & \cdot & \cdot & 1
\end{array}\right) \\
& \left(\begin{array}{cccc}
\cdot & 2 & \cdot & \cdot \\
\cdot & \cdot & \cdot & 1 \\
1 & \cdot & \cdot & .
\end{array}\right),\left(\begin{array}{cccc}
\cdot & 1 & \cdot & 1 \\
1 & \cdot & \cdot & \cdot \\
\cdot & 1 & . & .
\end{array}\right),\left(\begin{array}{cccc}
\cdot & 1 & \cdot & 1 \\
\cdot & 1 & \cdot & \cdot \\
1 & . & \cdot & \cdot
\end{array}\right)
\end{aligned}
$$

the fourth and the fifth matrices having 1134 as parkized word whereas the other ones yield 1234 .

It is interesting to observe that these algebras are nonunital. Indeed, (201) yields

Corollary 5.7. The element $\mathbf{J}_{n}=\mathbf{P}^{\left(1^{n}\right)}$ is a left unit for $*$, but not a right unit.

The description of $\mathbf{P}^{\pi^{\prime}} * \mathbf{P}^{\pi^{\prime \prime}}$ in terms of integer matrices being essentially identical to that of $S^{I} * S^{J}$ in $\mathbf{S y m}$, the same argument as in [7, proof of Proposition 5.2] shows that the splitting formula remains valid in $\mathbf{C Q S y m} \mathbf{m}_{n}$ :

Proposition 5.8. Let $\mu_{r}$ denote the $r$-fold product map from $\mathbf{C Q S y m}^{\otimes r}$ to $\mathbf{C Q S y m}, \Delta^{r}$ the $r$-fold coproduct with values in $\mathbf{C Q S y m}{ }^{\otimes r}$, and $*_{r}$ the internal product of the $r$-fold tensor product of algebras $\mathbf{C Q S y m}{ }^{\otimes r}$. Then, for $f_{1}, \ldots, f_{r}, g \in \mathbf{C Q S y m}$,

$$
\left(f_{1} \cdots f_{r}\right) * g=\mu_{r}\left[\left(f_{1} \otimes \cdots \otimes f_{r}\right) *_{r} \Delta^{r}(g)\right] .
$$

This is indeed the same formula as for internal product of $\mathbf{S y m}$, actually, an extension of it, since we have

COROLlary 5.9. The Hopf subalgebra of CQSym generated by the elements $\mathbf{J}_{n}$, which is isomorphic to $\mathbf{S y m}$ by $j: S_{n} \mapsto \mathbf{J}_{n}$, is stable under *, and thus also *-isomophic to Sym. Moreover, the map $f \mapsto f * \mathbf{J}_{n}$ is a projector onto $\mathbf{S y m}_{n}$, which is therefore a left $*$-ideal of $\mathbf{C Q S \mathbf { y m }} \mathbf{m}_{n}$.

More precisely, if $i<j<\cdots<r$ are the letters occurring in $\pi$, so that 
as a word $\pi=i^{m_{i}} j^{m_{j}} \ldots r^{m_{r}}$, then

$$
\mathbf{P}^{\pi} * \mathbf{J}_{n}=\mathbf{J}_{m_{i}} \mathbf{J}_{m_{j}} \cdots \mathbf{J}_{m_{r}} .
$$

It follows from Theorem 5.5 that the $\mathbf{R}_{\pi}$ are the pre-images of the ordinary ribbons under the projection $f \mapsto f * \mathbf{J}_{n}$ :

COROLlary 5.10. Let I be the composition obtained by discarding the zeros of the evaluation of a nondecreasing parking function $\pi$. Then

$$
\mathbf{R}_{\pi} * \mathbf{J}_{n}=j\left(R_{I}\right) \text {. }
$$

More precisely, if $I=\left(i_{1}, \ldots, i_{p}\right)$, this last element is equal to $\mathbf{R}_{1^{i_{1}} \bullet \cdots \bullet 1^{i_{p}}}$, that is, the Catalan ribbon indexed by the only nondecreasing word of evaluation $d(\pi)$.

The internal product of CQSym is dual to the coproduct $\delta f=f(X Y)$ on the commutative algebra CQSym, quotient of PQSym*. For example, we have

$$
\begin{gathered}
=\left(\mathcal{M}_{112}(X)+\mathcal{M}_{113}(X)\right)\left(\mathcal{M}_{111}(Y)+\mathcal{M}_{112}(Y)+\mathcal{M}_{113}(Y)+\mathcal{M}_{122}(Y)\right) \\
+\mathcal{M}_{111}(X) \mathcal{M}_{113}(Y), \\
\mathcal{M}_{112}(X Y)=\mathcal{M}_{111}(X) \mathcal{M}_{112}(Y)
\end{gathered}
$$

5.4.1. Cauchy kernel. Define the Cauchy kernel by

$$
K(X ; A)=\sum_{\mathbf{a} \in \mathrm{PF}} \mathbf{G}_{\mathbf{a}}(X) \mathbf{F}_{\mathbf{a}}(A)=\sum_{\pi} \mathcal{M}_{\pi}(X) \mathbf{P}^{\pi}(A) .
$$

Proposition 5.11. The kernel $K$ has the reproducing property

$$
K(X ; A) * K(Y ; A)=K(X Y ; A) .
$$

Proof.

$$
\begin{aligned}
\left\langle K(X) * K(Y), \mathcal{M}_{\pi}\right\rangle & =\sum_{\pi^{\prime}, \pi^{\prime \prime}} \mathcal{M}_{\pi^{\prime}}(X) \mathcal{M}_{\pi^{\prime \prime}}(Y)\left\langle\mathbf{P}^{\pi^{\prime}} * \mathbf{P}^{\pi^{\prime \prime}}, \mathcal{M}_{\pi}\right\rangle \\
& =\sum_{\pi^{\prime}, \pi^{\prime \prime}} \mathcal{M}_{\pi^{\prime}}(X) \mathcal{M}_{\pi^{\prime \prime}}(Y)\left\langle\mathbf{P}^{\pi^{\prime}} \otimes \mathbf{P}^{\pi^{\prime \prime}}, \Delta \mathcal{M}_{\pi}\right\rangle \\
& =\Delta \mathcal{M}_{\pi}(X, Y)=\mathcal{M}_{\pi}(X Y)
\end{aligned}
$$

\subsection{Compositions, Lagrange inversion, and $H_{n}(0)$}

5.5.1. Recall that nondecreasing parking functions (or noncrossing partitions) can be classified according to the factorization $\pi=\pi_{1} \bullet \cdots \bullet \pi_{r}$ into irreducible nondecreasing parking functions (or noncrossing partitions). Let $i_{k}:=\left|\pi_{k}\right|$ and $c(\pi):=\left(i_{1}, \ldots, i_{k}\right)$, regarded as a composition of $n$. 
We set

$$
\mathbf{V}^{I}:=\sum_{c(\pi)=I} \mathbf{P}^{\pi}
$$

considered as an element of PQSym. If one defines $\mathbf{V}_{n}=\mathbf{V}^{(n)}$, we have

$$
\mathbf{V}_{n}=\sum_{\mathbf{a} \in \mathrm{PPF}_{n}} \mathbf{F}_{\mathbf{a}}
$$

and

$$
\mathbf{V}^{I}=\mathbf{V}_{i_{1}} \cdots \mathbf{V}_{i_{r}}=\sum_{\mathbf{a} \in \mathrm{PPF}_{I}} \mathbf{F}_{\mathbf{a}}
$$

This can be reformulated as

$$
\sum_{\mathbf{a} \in \mathrm{PF}} \mathbf{F}_{\mathbf{a}}=\left(1-\sum_{\mathbf{b} \in \mathrm{PPF}} \mathbf{F}_{\mathbf{b}}\right)^{-1}
$$

which is the lift to PQSym of the well known identity

$$
\sum_{n \geq 0}(n+1)^{n-1} \frac{t^{n}}{n !}=\left(1-\sum_{n \geq 1}(n-1)^{n-1} \frac{t^{n}}{n !}\right)^{-1} .
$$

Indeed, the map $\mathbf{F}_{\mathbf{a}} \mapsto 1 / n !(n=|\mathbf{a}|)$ is a character of PQSym.

At this point, it is useful to observe that if $C(w)$ denotes the descent composition of a word $w$, the map

$$
\eta: \mathbf{F}_{\mathbf{a}} \mapsto F_{C(\mathbf{a})},
$$

which is a Hopf algebra morphism PQSym $\rightarrow$ QSym, maps $\mathbf{V}^{I}$ to the Frobenius characteristic of the underlying permutation representation of $\mathfrak{S}_{n}$ on $\mathrm{PPF}_{I}$ :

$$
\eta\left(\mathbf{V}^{I}\right)=\sum_{\mathbf{a} \in \mathrm{PPF}_{I}} \mathbf{F}_{C(\mathbf{a})}=\operatorname{ch}\left(\mathrm{PPF}_{I}\right)
$$

Indeed, if $V \subseteq A^{n}$ is any set of words invariant under the right action of $\mathfrak{S}_{n}$, the characteristic of the underlying permutation representation is always equal to $\sum_{w \in V} F_{C(w)}$. This is because $V$ splits as a disjoint union

$$
V=\bigsqcup_{\nu} A_{\nu}, \quad \text { where } \quad A_{\nu}=\left\{w \in A^{n} \mid \operatorname{Ev}(w)=\nu\right\}
$$

The characteristic of $A_{\nu}$ is clearly $h_{\nu}$, and it is well known that

$$
h_{\nu}=\sum_{w \in A_{\nu}} F_{C(w)} \text {. }
$$

Actually, each $\mathbb{C} A_{\nu}$ is also a projective $H_{n}(0)$-module with noncommutative characteristic $\operatorname{ch}\left(\mathbb{C} A_{\nu}\right)=S^{I}$, where $I=t(\nu)$. 
5.5.2. As a consequence, the number of parking functions of type $I$ with descent composition $J$ is equal to the scalar product of symmetric functions

$$
\left\langle r_{J}, f^{I}\right\rangle
$$

where $f^{I}=f_{i_{1}} \cdots f_{i_{r}}=\operatorname{ch}\left(\mathrm{PPF}_{I}\right)$ and $r_{J}$ is the ribbon Schur function. This extends Proposition 3.2(a) of [36]. Note that in particular, by inversion of

$$
\mathbf{F}_{\mathrm{PF}_{n}}:=\sum_{\mathbf{a} \in \mathrm{PF}_{n}} \mathbf{F}_{\mathbf{a}}=\sum_{I \models n} \mathbf{V}^{I}
$$

one obtains

$$
\mathbf{F}_{\mathrm{PPF}_{n}}=\sum_{I \models n}(-1)^{n-l(I)} \mathbf{F}_{\mathrm{PF}_{I}}
$$

where

$$
\mathrm{PF}_{I}:=\mathrm{PF}_{i_{1}} \uplus \ldots \cup \mathrm{PF}_{i_{r}} .
$$

These identities are easily visualized on the encoding of parking functions with skew Young diagrams as in [30] or in [9].

5.5.3. The transpose $\gamma^{*}$ of the map $\gamma$ defined in (193) is the map

$$
\text { ch : CQSym } \rightarrow \text { Sym, } \quad \mathbf{P}^{\pi} \mapsto S^{t(\pi)},
$$

which sends $\mathbf{P}^{\pi}$ to the characteristic noncommutative symmetric function of the natural projective $H_{n}(0)$-module with basis $\left\{\mathbf{a} \in \mathrm{PF}_{n} \mid \mathrm{NC}(\mathbf{a})=\pi\right\}$.

5.5.4. One can show that

$$
g:=\sum_{n \geq 0} g_{n}:=\sum_{n \geq 0} \operatorname{ch}\left(\mathbf{F}_{\mathrm{PF}_{n}}\right)=\sum_{I} \operatorname{ch}\left(\mathbf{V}^{I}\right)
$$

is the series obtained by applying the noncommutative Lagrange inversion formula of $[8,31]$ to the generating series of complete functions, i.e., $g$ is the unique solution of the equation

$$
g=1+S_{1} g+S_{2} g^{2}+\cdots=\sum_{n \geq 0} S_{n} g^{n} .
$$

Indeed, let $g$ be defined by (228) and set

$$
f:=\sum_{n \geq 1} f_{n}=\sum_{n \geq 1} \operatorname{ch}\left(\mathbf{F}_{\mathrm{PPF}_{n}}\right)=\sum_{n \geq 1} \operatorname{ch}\left(\mathbf{V}_{n}\right) .
$$

Recall that the prime nondecreasing parking functions of length $n$ are obtained by concatenating a 1 to the left of a nondecreasing parking function of length $n-1$. This gives a recurrence for $f_{n}$ and $g_{n}$. From each nondecreasing parking function with packed evaluation $J \vDash n-1$, contributing a term $S^{J}$ to $g_{n-1}$, we get a prime nondecreasing parking function with packed evaluation $I=\left(j_{1}+1, j_{2}, \ldots, j_{r}\right) \vDash n$, contributing a term $S^{I}:=\Omega S^{J}$ to $f_{n}$, 
where $\Omega$ is the linear operator incrementing the first part in the basis $S^{J}$ of Sym. Hence, $f_{n}=\Omega g_{n-1}$, and we have a system of two equations

$$
\left\{\begin{array}{l}
f=\Omega g, \\
g=(1-f)^{-1},
\end{array}\right.
$$

which, with the initial condition $g_{0}=1$, admits a unique solution: $f_{1}=$ $\Omega g_{0}=S^{1}, g_{1}=f_{1}, f_{2}=\Omega g_{1}=S^{2}, g_{2}=f_{2}+f^{11}=S^{2}+S^{11}, f_{3}=\Omega g_{2}=$ $S^{3}+S^{21}, g_{3}=f_{3}+f^{21}+f^{12}+f^{111}=S^{3}+2 S^{21}+S^{12}+S^{111}$, and so on.

But the unique solution of (229) satisfies

$$
\Omega g=S_{1}+S_{2} g+S_{3} g^{2}+\cdots
$$

and also

$$
1=g^{-1}+S_{1}+S_{2} g+S_{3} g^{2}+\cdots=g^{-1}+\Omega g
$$

so that if we set $f=\Omega g$, we solve (231) as well.

We remark that the commutative images of these equations give the $\mathfrak{S}_{n}$-characteristics of $\mathrm{PF}_{n}$ and $\mathrm{PPF}_{n}$, and that we have derived them from first principles, using only the multiplication rule of PQSym and the notion of a prime parking function.

5.5.5. The Hilbert series of $\mathbf{S Q S y m}$ revisited. It is also possible to obtain it by a character calculation, derived from the above considerations. If we decompose the noncommutative characteristic of the $H_{n}(0)$-module $\mathrm{CPF}_{n}$ into ribbons

$$
\operatorname{ch}\left(\mathbb{C P F}_{n}\right)=\sum_{I \models n} m_{I} R_{I},
$$

the number of hypoplactic classes of parking functions of length $n$ is

$$
\sum_{I \models n} m_{I} \text {. }
$$

Indeed, as already mentioned, if $V \subset A^{n}$ is any set of words which is a disjoint union of evaluation classes $A_{\nu}$, then $\mathbb{C} V$ is a projective $H_{n}(0)$ module since it is the direct sum $\bigoplus \mathbb{C} A_{\nu}$, where $\operatorname{ch}\left(\mathbb{C} A_{\nu}\right)=S^{I}$, with $I=$ $t(\nu)$.

Now, each $A_{\nu}$ is itself a disjoint union of hypoplactic classes

$$
A_{\nu, I}=\left\{w \in A_{\nu} \mid C\left(\operatorname{Std}(w)^{-1}\right)=I\right\},
$$

and each such class is the support of an indecomposable projective module

$$
\operatorname{ch}\left(A_{\nu, I}\right)=R_{I}
$$

By duality between the bases $F_{I}$ and $R_{I}$,

$$
\sum m_{I}=\left\langle\sum F_{I}, \operatorname{ch}\left(\mathbb{C P F}_{n}\right)\right\rangle
$$

and taking into account the identity [38] 


$$
\sum_{I} F_{I}=\frac{1}{2}\left[1+\prod_{i \geq 1} \frac{1+x_{i}}{1-x_{i}}\right]=1+\frac{1}{2} \sum_{n \geq 1} \sum_{k=0}^{n} e_{k} h_{n-k}
$$

we obtain

$$
\begin{aligned}
\operatorname{dim}\left(\mathbf{S Q S y m}_{n}\right) & =\left\langle\sum_{I \models n} F_{I}, \mathbf{c h}\left(\mathbf{F}_{\mathrm{PF}_{n}}\right)\right\rangle \\
& =\left\langle\frac{1}{2} \sum_{k=0}^{n} e_{k} h_{n-k}, \frac{1}{n+1} h_{n}((n+1) X)\right\rangle \\
& =\frac{1}{2 n+2} \sum_{k=0}^{n}\left(\begin{array}{c}
n+1 \\
k
\end{array}\right)\left(\begin{array}{c}
2 n-k \\
n-k
\end{array}\right)=s_{n} .
\end{aligned}
$$

\section{A HOPF ALGEBRA OF SEGMENTED COMPOSITIONS}

6.1. Segmented compositions. Define a segmented composition as a finite sequence of positive integers, separated by vertical bars or commas, e.g., $(2,1|2| 1,2)$.

The number of segmented compositions having the same underlying composition is obviously $2^{l-1}$ where $l$ is the length of the composition, so that the total number of segmented compositions with sum $n$ is $3^{n-1}$ since $(1+2)^{n-1}=3^{n-1}$.

\subsection{A Hopf subalgebra of SQSym*}

6.2.1. Hypoplactic packed words. Let $A=\left\{a_{1}<a_{2}<\cdots\right\}$ be an infinite totally ordered alphabet. The packed word $u=\operatorname{pack}(w)$ associated with a word $w \in A^{*}$ is obtained by the following process. If $b_{1}<\cdots<b_{r}$ are the letters occurring in $w$, then $u$ is the image of $w$ under the homomorphism $b_{i} \mapsto a_{i}$.

A word $u$ is said to be packed if $\operatorname{pack}(u)=u$. We denote by PW the set of packed words.

Let us consider packed quasi-ribbons, that is, quasi-ribbons that are packed words. For example, the word 11324355 is a packed quasi-ribbon word since it is the reading of the following quasi-ribbon:

$$
\begin{array}{|l|l|l|l|l|l|l|l|l|l|l|l|l|l}
\hline & 1 & 2 & & & \\
& & 3 & 3 & & \\
\hline
\end{array}
$$

These objects are in bijection with segmented compositions. Indeed, start from a packed quasi-ribbon $\mathbf{q}$ and write the evaluation vector $\mathbf{I}$ of $\mathbf{q}$, putting a separator between $\mathbf{I}_{i}$ and $\mathbf{I}_{i+1}$ iff $i$ and $i+1$ are not is the same row of q. For example, the segmented composition corresponding to the quasiribbon of (241) is $21|2| 12$. This element will be denoted by $\operatorname{ps}(\mathbf{q})$. The reverse 
bijection consists in writing the unique nondecreasing word with evaluation I and put the letters $i+1$ on the row next to the row of the letters of $i$ iff $i$ and $i+1$ are separated by $\mid$ in $\mathbf{I}$.

EXAMPLE 6.1. For $n=2$, we have three packed quasi-ribbons

$$
11, \quad 12, \quad 1 \mid 2,
$$

For $n=3$, we have nine packed quasi-ribbons

$$
111, \quad 112, \quad 11|2, \quad 122, \quad 1| 22, \quad 123, \quad 1|23, \quad 12| 3, \quad 1|2| 3 .
$$

respectively encoded as the nine segmented compositions

$$
3, \quad 21, \quad 2|1, \quad 12, \quad 1| 2, \quad 111, \quad 1|11, \quad 11| 1, \quad 1|1| 1 .
$$

In the following, we will identify packed quasi-ribbons and their encodings as segmented compositions.

6.2.2. A Hopf subalgebra of SQSym*. Let us denote by $\mathrm{P}(w)$ the hypoplactic $P$-symbol of a word $w$ (its quasi-ribbon). The $P$-symbols of packed words are therefore packed quasi-ribbons.

For each packed quasi-ribbon $\mathbf{I}$, define

$$
P_{\mathbf{I}}:=\mathbf{P}_{\text {unp }(\mathbf{I})} \quad \text { and } \quad Q_{\mathbf{I}}:=\sum_{\mathrm{ps}(\mathbf{q})=\mathbf{I}} \mathbf{Q}_{\mathbf{q}} \in \mathbf{S Q S y m}^{*} .
$$

where $\operatorname{unp}(\mathbf{q})$ is the maximal parking quasi-ribbon for the lexicographic order of evaluation $\mathbf{I}$.

For example,

$$
\begin{array}{rlrl}
P_{11 \mid 2} & =\mathbf{P}_{12 \mid 33}, & P_{112}=\mathbf{P}_{1233}, \\
Q_{12 \mid 1} & =\mathbf{Q}_{122 \mid 3}+\mathbf{Q}_{122 \mid 4}, & Q_{121} & =\mathbf{Q}_{1223}+\mathbf{Q}_{1224}, \\
Q_{12 \mid 21} & =\mathbf{Q}_{122 \mid 334}+\mathbf{Q}_{122 \mid 335}+\mathbf{Q}_{122 \mid 336}+\mathbf{Q}_{122 \mid 445}+\mathbf{Q}_{122 \mid 446} .
\end{array}
$$

Theorem 6.2. The $P_{\mathbf{I}}$ span a Hopf subalgebra $\mathbf{S C Q S y m}$ of SQSym. This subalgebra is also the quotient of $\mathbf{S C Q S y m}$ by the relations $\mathbf{P}_{\mathbf{q}}=\mathbf{P}_{\mathbf{q}^{\prime}}$ if $w$ and $w^{\prime}$ have the same packed word.

The $Q_{\mathbf{I}}$ span a Hopf subalgebra $\mathbf{S C Q S y m} *$ of $\mathbf{S Q S y m}{ }^{*}$, and

$$
\operatorname{dim} \mathbf{S C Q S y m}_{n}=3^{n-1} \quad \text { for } n \geq 1 \text {. }
$$

Proof. The product and coproduct rules of the $\mathbf{P}$ of SQSym imply that the $P$ of SQSym* span a Hopf subalgebra of SQSym. It is also obvious that both operations are compatible with the relations $\mathbf{P}_{\mathbf{q}}=\mathbf{P}_{\mathbf{q}^{\prime}}$ if $\mathbf{q}$ and $\mathbf{q}^{\prime}$ have same packed word, so that SCQSym is a Hopf quotient of SQSym. Then, by the standard argument, the $Q$ are a basis of SCQSym*.

The dimension of $\mathbf{S C Q S y m}_{n}$ is given by the number of segmented compositions, that is, $3^{n-1}$.

To describe the product and coproduct rules of both bases, we need a new operation on segmented compositions. 
Recall that the product of the monomial basis $M_{I}$ on QSym is defined with the augmented shuffle of two compositions recursively defined as

$$
\left(I_{1}, I^{\prime}\right) \uplus\left(J_{1}, J^{\prime}\right)=I_{1}\left(I^{\prime} \uplus\left(J_{1}, J^{\prime}\right)\right)+J_{1}\left(\left(I_{1}, I^{\prime}\right) \uplus J^{\prime}\right)+I_{1}+J_{1}\left(I^{\prime} \uplus J^{\prime}\right),
$$

with the extra condition $I^{\prime} \uplus \epsilon=\epsilon \uplus I^{\prime}$ where $\epsilon$ is the empty word.

This construction is generalized to SCQSym as follows: the augmented shuffle $\mathbf{I}^{\prime} \uplus \mathbf{I}^{\prime \prime}$ of two segmented compositions is obtained from the usual augmented shuffle $I^{\prime} \uplus I^{\prime \prime}$ of their underlying compositions by inserting bars between two blocks $I_{k}$ and $I_{k+1}$ of a composition $I$ iff

- $I_{k}$ and $I_{k+1}$ both contain elements coming from $\mathbf{I}^{\prime}$ and those elements were separated by a bar,

- $I_{k}$ and $I_{k+1}$ both contain elements coming from $\mathbf{I}^{\prime \prime}$ and those elements were separated by a bar,

- $I_{k}$ contains an element coming from $\mathbf{I}^{\prime \prime}$ and $I_{k+1}$ contains an element coming from $\mathbf{I}^{\prime}$.

For example,

$$
\begin{gathered}
1 \uplus 2|1=12| 1+3|1+2| 11+2|2+2| 1 \mid 1, \\
1 \uplus 21=121+31+2|11+2| 2+21 \mid 1 .
\end{gathered}
$$

TheOREm 6.3. The product and coproduct rules for the $P_{\mathbf{I}}$ and the $Q_{\mathbf{I}}$ are

$$
\begin{aligned}
P_{\mathbf{I}^{\prime}} P_{\mathbf{I}^{\prime \prime}} & =P_{\mathbf{I}^{\prime} \mid \mathbf{I}^{\prime \prime}}+P_{\mathbf{I}^{\prime} \mathbf{I}^{\prime \prime}}, \\
\Delta P_{\mathbf{I}} & =\sum_{\mathbf{I} \in \mathbf{I}^{\prime} \uplus \mathbf{I}^{\prime \prime}} \mathbf{P}_{\mathbf{I}^{\prime}} \otimes \mathbf{P}_{\mathbf{I}^{\prime \prime}}, \\
Q_{\mathbf{I}^{\prime}} Q_{\mathbf{I}^{\prime \prime}} & =\sum_{\mathbf{I} \in \mathbf{I}^{\prime} \uplus \mathbf{I}^{\prime \prime}} Q_{\mathbf{I}}, \\
\Delta Q_{\mathbf{I}} & =\sum_{\mathbf{I}=\mathbf{I}^{\prime} \cdot \mathbf{I}^{\prime \prime} \text { or } \mathbf{I}=\mathbf{I}^{\prime} \mid \mathbf{I}^{\prime \prime}} Q_{\mathbf{I}^{\prime}} \otimes Q_{\mathbf{I}^{\prime \prime}} .
\end{aligned}
$$

Proof. The product of two $P$ of SCQSym directly comes from the product of two $\mathbf{P}$ of SQSym. The coproduct of a $Q$ then follows by duality. The shifted shuffle of two compositions obviously gives all the possible evaluations of the convolution of two parking functions of the given evaluations. Finally, the rules to place the bars correspond to the different cases where there is an $i$ to the right of an $i+1$ in one of the resulting parking functions.

For example,

$$
\begin{gathered}
P_{12 \mid 1} P_{2 \mid 11}=P_{12|12| 11}+P_{12|1| 2 \mid 11} \\
\Delta P_{12 \mid 1}=1 \otimes P_{12 \mid 1}+P_{1} \otimes\left(P_{12}+P_{2 \mid 1}\right)+P_{11} \otimes\left(P_{1 \mid 1}+P_{2}\right) \\
+P_{1 \mid 1} \otimes P_{2}+\left(P_{111}+P_{11 \mid 1}\right) \otimes P_{1}+P_{12 \mid 1} \otimes 1
\end{gathered}
$$




$$
\begin{gathered}
Q_{1} Q_{2 \mid 1}=Q_{3 \mid 1}+Q_{12 \mid 1}+Q_{2 \mid 2}+Q_{2|1| 1}+Q_{2 \mid 11} \\
Q_{1} Q_{11 \mid 1}=Q_{111 \mid 1}+Q_{21 \mid 1}+Q_{1|11| 1}+Q_{1|2| 1}+Q_{11 \mid 11}+Q_{11 \mid 2}+Q_{11|1| 1} \\
Q_{1 \mid 1} Q_{1 \mid 1}=2 Q_{1|11| 1}+Q_{1|1| 11}+Q_{11 \mid 11}+Q_{11|1| 1}+Q_{1|1| 1 \mid 1} \\
+Q_{2 \mid 11}+Q_{2|1| 1}+Q_{2 \mid 2}+2 Q_{1|2| 1}+Q_{1|1| 2}+Q_{11 \mid 2} \\
\Delta Q_{12 \mid 1}=1 \otimes Q_{12 \mid 1}+Q_{1} \otimes Q_{2 \mid 1}+Q_{12} \otimes Q_{1}+Q_{12 \mid 1} \otimes 1 .
\end{gathered}
$$

6.3. Algebraic structure of SCQSym and SCQSym*. The algebra SCQSym* is not free for exactly the same reason $\mathbf{S Q S y m} *$ is not: one has the relation

$$
Q_{1}\left(Q_{2}+Q_{11}\right)=\left(Q_{2}+Q_{11}\right) Q_{1} .
$$

Let us now move to SCQSym.

Since SCQSym is the subalgebra of SQSym spanned by the parking quasi-ribbons that are maximally unpacked, and since SQSym is free, SCQSym is automatically free and generated by the maximal elements of PQS. For example, the generators of SCQSym for $n \leq 4$ are

$$
\begin{aligned}
& \{1\}, \quad\{11,1 \mid 2\}, \quad\{111,11|3,1| 22,1|2| 3\}, \\
& \{1111,111|4,11| 33,11|3| 4,1|222,1| 22|4,1| 2|33,1| 2|3| 4\},
\end{aligned}
$$

that can be rewritten on segmented compositions as

$$
\begin{aligned}
& \{1\}, \quad\{2,1 \mid 1\}, \quad\{3,2|1,1| 2,1|1| 1\}, \\
& \{4,3|1,2| 2,2|1| 1,1|3,1| 2|1,1| 1|2,1| 1|1| 1\},
\end{aligned}
$$

By the same argument on generating series as in SQSym, one finds that there are $2^{n-1}$ generators of SCQSym of degree $n$. And indeed, these generators are in natural bijection with compositions of $n$ since they have separators between all elements.

The next proposition summarizes the structures of SCQSym and SCQSym*.

Proposition 6.4. The algebra SCQSym is a Hopf algebra of dimension $3^{n-1}$. It is not self-dual since $\mathbf{S C Q S y m}$ is free as an algebra whereas SCQSym* is not. Moreover, SCQSym is free over a graded alphabet labelled by all compositions.

6.4. Primitive Lie algebras of SCQSym*. Since SCQSym* contains QSym as a subalgebra, its primitive Lie algebra cannot be free and one easily finds that

$$
\left[Q_{1}, Q_{12}-Q_{1 \mid 2}+Q_{11}\right]=0
$$


6.5. A quasi-ribbon basis of SCQSym*. The elements $Q_{\mathbf{I}}$ are segmented analogs of the basis $\left(M_{I}\right)$ of QSym. So we can define analogs of the $\left(F_{I}\right)$ of QSym in the same way as we did in SQSym*.

Recall that the refinement order denoted by $\succeq$ on compositions is such that $I=\left(i_{1}, \ldots, i_{k}\right) \succeq J=\left(j_{1}, \ldots, j_{l}\right)$ iff $\left\{i_{1}, i_{1}+i_{2}, \ldots, i_{1}+\cdots+i_{k}\right\}$ contains $\left\{j_{1}, j_{1}+j_{2}, \ldots, j_{1}+\cdots+j_{l}\right\}$. In this case, we say that $I$ is finer than $J$. For example, $(2,1,2,3,1,2) \succeq(3,2,6)$.

Let $\mathbf{I}=\left(I_{1}|\cdots| I_{r}\right)$ and let

$$
F_{\mathbf{I}}:=\sum_{\mathbf{I}^{\prime}} Q_{\mathbf{I}^{\prime}}
$$

where the sum is taken over sequences of compositions $\left(I_{1}^{\prime}, \ldots, I_{r}^{\prime}\right)$ where $I_{k}^{\prime}$ is finer than $I_{k}$. For example,

$$
F_{2 \mid 2}=Q_{11 \mid 11}+Q_{2 \mid 1}+Q_{11 \mid 2}+Q_{2 \mid 2} .
$$

By a triangularity argument, we have

TheOREM 6.5. The $F_{\mathbf{I}}$ form a basis of SCQSym* .

The basis $F_{\mathbf{I}}$ satisfies a product formula similar to the $F_{I}$ of QSym (whence the choice of notation). To state it, we need an analogue of the shifted shuffle.

A segmented permutation is a permutation with separators. The descent composition $C(\alpha)$ of a segmented permutation $\alpha$ is a segmented composition: it is the sequence of descent compositions of the blocks of $\alpha$ separated by bars.

For example, $\alpha=248|517| 3$ is a segmented permutation whose descent composition is $(3|12| 1)$.

The shifted shuffle $\alpha \mathbb{U} \beta$ of two segmented permutations is obtained from the usual shifted shuffle $\sigma \in \tau$ of the underlying permutations $\sigma$ and $\tau$ by inserting bars:

- after each descent which was originally followed by a bar in $\alpha$ or in the shift of $\beta$,

- after each descent created by the shuffling process.

For example,

$$
2|1 \cup 21=2| 143+24|13+243| 1+4|2| 13+4|23| 1+43|2| 1 .
$$

TheOREM 6.6. Let $\mathbf{I}^{\prime}$ and $\mathbf{I}^{\prime \prime}$ be two segmented compositions and let $\alpha$ and $\beta$ be any two segmented permutations whose descent compositions are respectively $\mathbf{I}^{\prime}$ and $\mathbf{I}^{\prime \prime}$. Then

$$
F_{\mathbf{I}^{\prime}} F_{\mathbf{I}^{\prime \prime}}=\sum_{\mathbf{I}} F_{\mathbf{I}}
$$

where the sum runs over the descent compositions of the segmented permutations $\gamma$ occurring in $\alpha \uplus \beta$. 
Proof. The product of the $Q$ can be easily rewritten in terms of segmented permutations as follows: associate with two segmented compositions $\mathbf{I}^{\prime}$ and $\mathbf{I}^{\prime \prime}$ two segmented permutations $\alpha$ and $\beta$ such that $C(\alpha)=\mathbf{I}^{\prime}$ and $C(\beta)=\mathbf{I}^{\prime \prime}$. Consider the elements in the shifted shuffle $\alpha \in \beta$ such that two elements in increasing order of $\alpha$ (resp. $\beta$ ) not separated by a bar have no $\beta$ (resp. $\alpha$ ) between them. From all those elements, build the set of all segmented permutations with at least those bars and at most new bars between the elements of $\alpha$ and the elements of $\beta$. For all those segmented permutations, compute first their descent compositions and then remove the bars added lately. The set of the descent compositions obtained by this process corresponds to the product $Q_{\alpha} Q_{\beta}$.

Express both $F$ in the $Q$ basis and group the terms in their product where the letters of $\alpha$ have been inserted at the same place. By construction, the lexicographically minimum element $s$ in each group with the smallest number of bars belongs to $\alpha \uplus \beta$. Now, given the product rule of the $Q$, we have all elements obtained from $s$ by adding any number of bars, thus $F_{s}$.

For example,

$$
\begin{aligned}
F_{1} F_{1|| 1} & =F_{21 \mid 1}+F_{1|2| 1}+F_{11 \mid 2}+F_{11|1| 1}, \\
F_{1} F_{2 \mid 1} & =F_{3 \mid 1}+F_{1|2| 1}+F_{2 \mid 2}+F_{2|1| 1} .
\end{aligned}
$$

THEOREM 6.7. Let $\mathbf{I}$ be a segmented composition. Then

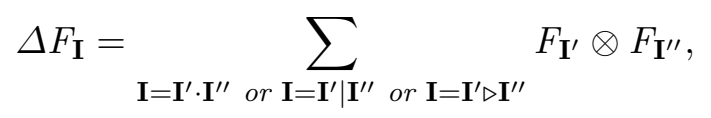

where $\left(I_{1}, \ldots, I_{k}\right) \triangleright\left(I_{1}^{\prime}, \ldots, I_{l}^{\prime}\right)$ denotes the segmented composition $\left(I_{1}, \ldots\right.$, $\left.I_{k-1}, I_{k} \triangleright^{\prime} I_{1}^{\prime}, I_{2}^{\prime}, \ldots, I_{l}^{\prime}\right)$ where $\left(i_{1}, \ldots, i_{k}\right) \triangleright^{\prime}\left(j_{1}, \ldots, j_{l}\right)=\left(i_{1}, \ldots, i_{k-1}, i_{k}+j_{1}\right.$, $\left.j_{2}, \ldots, j_{l}\right)$.

Proof. This result will follow by duality from the considerations in the forthcoming section.

6.6. A ribbon basis of SCQSym. Let $\left(R_{\mathbf{I}}\right)$ be the dual basis of $\left(F_{\mathbf{I}}\right)$. Then we have

Proposition 6.8. The $\left(R_{\mathbf{I}}\right)$ are a basis of $\mathbf{S C Q S y m}$ related to the $P$ by

$$
P_{\mathbf{I}}=: \sum_{\mathbf{I}^{\prime}} R_{\mathbf{I}^{\prime}}
$$

where the sum is taken over sequences of segmented compositions $\left(I_{1}^{\prime}|\ldots| I_{r}^{\prime}\right)$ where $I_{k}$ is finer than $I_{k}^{\prime}$.

Since SCQSym is a subalgebra of SQSym such that the image of $P_{\mathbf{I}}$ is $\mathbf{P}_{\mathbf{q}}$, given both orders on parking quasi-ribbons and on segmented compositions, the image of $R_{\mathbf{I}}$ is $\mathbf{R}_{\mathbf{q}}$. This remark immediately proves the product 
rule of the $R$, whereas its coproduct rule comes from the duality between the $R$ and the $F$.

THEOREM 6.9. The product and coproduct rules of $R$ are

$$
\begin{aligned}
R_{\mathbf{I}^{\prime}} \cdot R_{\mathbf{I}^{\prime \prime}} & =R_{\mathbf{I}^{\prime} \cdot \mathbf{I}^{\prime \prime}}+R_{\mathbf{I}^{\prime} \mid \mathbf{I}^{\prime \prime}}+R_{\mathbf{I}^{\prime} \triangleright \mathbf{I}^{\prime \prime}}, \\
\Delta R_{\mathbf{I}} & =\sum_{\mathbf{I} \in \mathbf{I}^{\prime} \uplus \mathbf{I}^{\prime \prime}} R_{\mathbf{I}^{\prime}} \otimes R_{\mathbf{I}^{\prime \prime}} .
\end{aligned}
$$

From (274), we see that SCQSym is the free cubical trialgebra on one generator (see [22]).

\section{APPENDIX}

7.1. Relations with free probability theory. The free cumulants $R_{n}$ of a probability measure $\mu$ on $\mathbb{R}$ are defined (see, e.g., [35]) by means of the generating series of its moments $M_{n}$,

$$
G_{\mu}(z):=\int_{\mathbb{R}} \frac{\mu(d x)}{z-x}=z^{-1}+\sum_{n \geq 1} M_{n} z^{-n-1}
$$

as the coefficients of its compositional inverse

$$
K_{\mu}(z):=G_{\mu}(z)^{\langle-1\rangle}=z^{-1}+\sum_{n \geq 1} R_{n} z^{n-1} .
$$

It is in general instructive to interpret the coefficients of a formal power series as the specializations of the elements of some generating family of the algebra of symmetric functions. In this context, it is the interpretation

$$
M_{n}=\phi\left(h_{n}\right)=h_{n}(A)
$$

that is relevant. Indeed, the process of functional inversion (Lagrange inversion) admits a simple expression within this formalism (see [23, Ex. 24, p. 35]). If the symmetric functions $h_{n}^{*}$ are defined by the equations

$$
u=t H(t) \Leftrightarrow t=u H^{*}(u)
$$

where $H(t):=\sum_{n \geq 0} h_{n} t^{n}, H^{*}(u):=\sum_{n \geq 0} h_{n}^{*} u^{n}$, then, in the $\lambda$-ring notation,

$$
h_{n}^{*}(X)=\frac{1}{n+1}(-1)^{n} e_{n}((n+1) X):=\frac{1}{n+1}\left[t^{n}\right] E(-t)^{n+1}
$$

where $E(t)$ is defined by $E(t) H(t)=1$. This defines an involution $f \mapsto f^{*}$ of the ring of symmetric functions.

Now, if one sets $M_{n}=h_{n}(A)$ as above, then

$$
\begin{aligned}
G_{\mu}(z)= & z^{-1} H\left(z^{-1}\right)=u \\
& \Leftrightarrow z=K_{\mu}(u)=\frac{1}{u} E^{*}(-u)=u^{-1}+\sum_{n \geq 1}(-1)^{n} e_{n}^{*} u^{n-1} .
\end{aligned}
$$


Hence,

$$
R_{n}=(-1)^{n} e_{n}^{*}(A)
$$

7.2. An exercise on permutation representations. It follows immediately from the explicit formula (see [23, p. 35])

$$
-e_{n}^{*}=\frac{1}{n-1} \sum_{\lambda \vdash n}\left(\begin{array}{c}
n-1 \\
l(\lambda)
\end{array}\right)\left(\begin{array}{c}
l(\lambda) \\
m_{1}, m_{2}, \ldots, m_{n}
\end{array}\right) e_{\lambda}
$$

(where $\lambda=1^{m_{1}} 2^{m_{2}} \cdots n^{m_{n}}$ ) that $-e_{n}^{*}$ is Schur positive. Clearly, $-e_{n}^{*}$ is the Frobenius characteristic of a permutation representation $\Pi_{n}$, twisted by the sign character. Let us set

$$
(-1)^{n-1} R_{n}=-e_{n}^{*}=: \omega\left(f_{n}\right)
$$

so that

$$
f_{n}:=\sum_{\lambda \vdash n} \frac{1}{n-1}\left(\begin{array}{c}
n-1 \\
l(\lambda)
\end{array}\right)\left(\begin{array}{c}
l(\lambda) \\
m_{1}, m_{2}, \ldots, m_{n}
\end{array}\right) h_{\lambda}
$$

and $f_{n}$ is the character of $\Pi_{n}$.

The problem of constructing such a representation had been raised by Kerov in 1995 . We shall see that $\Pi_{n}$ corresponds to prime parking functions. We note that our construction of $\Pi_{n}$ is merely a variation on previously known results (see in particular $[18,30]$ ). However, since this is this precise version of the question that led us to the Hopf algebra of parking functions and some of its properties, we decided to include its discussion.

\subsection{Solution of the exercise}

Proposition 7.1. The Frobenius characteristic of the permutation representation of $\mathrm{PPF}_{n}$ is $f_{n}$.

Proof. We first show that the number of nondecreasing prime parking functions whose reordered evaluation is a given partition $\lambda$ is equal to

$$
\frac{1}{n-1}\left(\begin{array}{c}
n-1 \\
l(\lambda)
\end{array}\right)\left(\begin{array}{c}
l(\lambda) \\
m_{1}, m_{2}, \ldots, m_{n}
\end{array}\right)
$$

where $\lambda=1^{m_{1}} 2^{m_{2}} \cdots n^{m_{n}}$ (see $\left.(285)\right)$. Indeed, this number corresponds to the number of ways of putting the $\lambda_{i}$ over $n-1$ places in a circle. For such a placement $P$, number in all possible clockwise ways the places of the circle and consider the $n-1$ nondecreasing words $i^{c_{i}}$ where $c_{i}$ is the content of place number $i$. Then, by [5], exactly one of those words is a prime parking function. 
For example, for $\lambda=(3,2,2)$, there are ten possible circles. Consider the circle where the 3 is followed by one empty place, a 2, two empty places, and the last 2. Then the six nondecreasing words are:

$$
\text { 1113366, 2255666, 1144555, 3344466, 2233355, } 1122244 \text {. }
$$

Now, since all permutations of a nondecreasing prime parking function are parking functions, the Frobenius characteristic of the permutation representation of this set of words is $h_{\lambda}$. It then easily follows that

$$
\operatorname{ch}\left(\mathrm{PPF}_{n}\right)=f_{n},
$$

so that $\Pi_{n}$ can be identified with $\mathrm{PPF}_{n}$, as claimed before.

\section{References}

[1] M. Aguiar, N. Bergeron, and F. Sottile, Combinatorial Hopf algebras and generalized Dehn-Sommerville relations, Compos. Math. 142 (2006), 1-30.

[2] C. Athanasiadis, A simple bijection for regions of the Shi arrangement of hyperplanes, Discrete Math. 204 (1999), 27-39.

[3] P. Biane, Parking functions of types $A$ and B, Electron. J. Combin. 9 (2002), no. 7.

[4] G. Duchamp, F. Hivert, and J.-Y. Thibon, Noncommutative symmetric functions VI: free quasi-symmetric functions and related algebras, Internat. J. Algebra Comput. 12 (2002), 671-717.

[5] A. Dvoretzky and T. Motzkin, A problem of arrangements, Duke Math. J. 14 (1947), 303-313.

[6] L. Foissy, Bidendriform bialgebras, trees, and free quasi-symmetric functions, ArXiv math.RA/0505207.

[7] I. M. Gelfand, D. Krob, A. Lascoux, B. Leclerc, V. S. Retakh, and J.-Y. Thibon, Noncommutative symmetric functions, Adv. Math. 112 (1995), 218-348.

[8] I. Gessel, Noncommutative generalization and q-analog of the Lagrange inversion formula, Trans. Amer. Math. Soc. 257 (1980), 455-482.

[9] J. Haglund, M. Haiman, N. Loehr, J. B. Remmel, and A. Ulyanov, A combinatorial formula for the character of the diagonal coinvariants, Duke Math. J. 126 (2005), 195-232.

[10] M. Haiman, Conjectures on the quotient ring by diagonal invariants, J. Algebra Combin. 3 (1994), 17-76.

[11] F. Hivert, Combinatoire des fonctions quasi-symétriques, Thèse de Doctorat, MarneLa-Vallée, 1999.

[12] F. Hivert, J.-C. Novelli, and J.-Y. Thibon, The algebra of binary search trees, Theoret. Comput. Sci. 339 (2005), 129-165.

[13] - , - - - Commutative Hopf algebras of permutations and trees, preprint, 2005, ArXiv math.CO/0502456.

[14] F. Hivert and N. Thiéry, MuPAD-Combinat, an open-source package for research in algebraic combinatorics, Sém. Lothar. Combin. 51 (2004), 70 pp. (electronic).

[15] R. Holtkamp, On Hopf algebra structures over free operads, preprint, 2005, ArXiv math.RA/0504531.

[16] L. H. Kalikow, Enumeration of parking functions, allowable permutation pairs, and labelled trees, PhD Thesis, Brandeis Univ., 1999. 
[17] D. Krob and J.-Y. Thibon, Noncommutative symmetric functions IV: Quantum linear groups and Hecke algebras at $q=0$, J. Algebraic Combin. 6 (1997), 339-376.

[18] C. Lenart, Lagrange inversion and Schur functions, ibid. 11 (2000), 69-78.

[19] J.-L. Loday, Dialgebras, in: Dialgebras and Related Operads, Lecture Notes in Math. 1763, Springer, Berlin, 2001, 7-66.

[20] - , Parking functions and triangulation of the associahedron, preprint, ArXiv math.AT/0510380.

[21] J.-L. Loday and M. O. Ronco, Hopf algebra of the planar binary trees, Adv. Math. 139 (1998), 293-309.

[22] - - -, Trialgebras and families of polytopes, Contemp. Math. 346, Amer. Math. Soc., 2004, 369-398.

[23] I. G. Macdonald, Symmetric Functions and Hall Polynomials, 2nd ed., Oxford Univ. Press, 1995.

[24] C. Malvenuto and C. Reutenauer, Duality between quasi-symmetric functions and the Solomon descent algebra, J. Algebra 177 (1995), 967-982.

[25] T. V. Narayana, Lattice Path Combinatorics with Statistical Applications, Univ. Toronto Press, 1979.

[26] J.-C. Novelli, On the hypoplactic monoid, Discrete Math. 217 (2000), 315-336.

[27] J.-C. Novelli and J.-Y. Thibon, A Hopf algebra of parking functions, in: Proc. FPSAC'04, Vancouver, to appear.

[28] —, -, Parking functions and descent algebras, Ann. Combin., to appear.

[29] - , - Polynomial realizations of some trialgebras, in preparation.

[30] I. Pak and A. Postnikov, Enumeration of trees and one amazing representation of $S_{n}$, in: Proc. FPSAC'96 Conf. (Minneapolis, MN, 1996), 385-389.

[31] I. Pak, A. Postnikov, and V. S. Retakh, Noncommutative Lagrange theorem and inversion polynomials, talk at FPSAC'95, Marne-la-Vallée; preprint available at http://www-math.mit.edu/ ${ }^{\sim}$ pak/research.html.

[32] S. Poirier et C. Reutenauer, Algèbre de Hopf des tableaux, Ann. Sci. Math. Québec 19 (1995), 79-90.

[33] C. Reutenauer, Free Lie Algebras, Oxford Univ. Press, 1993.

[34] N. J. A. Sloane, The On-Line Encyclopedia of Integer Sequences, http://www.research. att.com $/{ }^{\sim}$ njas/sequences/.

[35] R. Speicher, Multiplicative functions on the lattice on non-crossing partitions and free convolution, Math. Ann. 298 (1994), 141-159.

[36] R. P. Stanley, Parking functions and noncrossing partitions, Electron. J. Combin. 4 (1997), no. 2.

[37] —, Enumerative Combinatorics, Vol. 2, Cambridge Univ. Press, 1999.

[38] B. C. V. Ung, Combinatorial identities for series of quasi-symmetric functions, in: FPSAC 98 (Toronto, 1998).

Institut Gaspard Monge

Université de Marne-la-Vallée

5 Boulevard Descartes

Champs-sur-Marne

77454 Marne-la-Vallée Cedex 2, France

E-mail: novelli@univ-mlv.fr jyt@univ-mlv.fr 